\title{
POINTWISE ERROR ESTIMATES OF FINITE ELEMENT APPROXIMATIONS TO THE STOKES PROBLEM ON CONVEX POLYHEDRA
}

\author{
J. GUZMÁN AND D. LEYKEKHMAN
}

\begin{abstract}
The aim of the paper is to show the stability of the finite element solution for the Stokes system in $W_{\infty}^{1}$ norm on general convex polyhedral domain. In contrast to previously known results, $W_{r}^{2}$ regularity for $r>3$, which does not hold for general convex polyhedral domains, is not required. The argument uses recently available sharp Hölder pointwise estimates of the corresponding Green's matrix together with novel local energy error estimates, which do not involve an error of the pressure in a weaker norm.
\end{abstract}

\section{INTRODUCTION}

Consider the following Stokes problem on a convex polyhedral domain $\Omega \subset \mathbb{R}^{3}$,

$$
\begin{aligned}
-\Delta \vec{u}+\nabla p=\vec{f} & \text { in } \Omega, \\
\nabla \cdot \vec{u}=0 & \text { in } \Omega, \\
\vec{u}=\overrightarrow{0} & \text { on } \partial \Omega .
\end{aligned}
$$

Here $\vec{u}=\left(u_{1}, u_{2}, u_{3}\right)$ represents the velocity of the fluid, $p \in L^{2}(\Omega)$ the pressure, and $\vec{f}=\left(f_{1}, f_{2}, f_{3}\right)$ is a smooth external force vector function. The solution $p \in L^{2}(\Omega)$ is unique up to a constant. Our work is motivated by [13], where the stability of the finite element solution, namely

$$
\left\|\nabla \vec{u}_{h}\right\|_{L^{\infty}(\Omega)}+\left\|p_{h}\right\|_{L^{\infty}(\Omega)} \leq C\left(\|\nabla \vec{u}\|_{L^{\infty}(\Omega)}+\|p\|_{L^{\infty}(\Omega)}\right)
$$

was derived under the regularity assumptions $\vec{u} \in W_{r}^{2}(\Omega)^{3}$ and $p \in W_{r}^{1}(\Omega)$, for some $r>3$. This result was an important improvement over previous results where the constant $C$ depended on $|\log h|$ (cf. [9]).

However, the drawback of the result in [13] is the assumption of $W_{r}^{2}(\Omega)^{3}$ regularity for some $r>3$. The standard regularity results (cf. 4]) for general convex polyhedral domains only give $\vec{u} \in H^{2}(\Omega)^{3}$ and $p \in H^{1}(\Omega)$, and in order to guarantee $\vec{u} \in W_{r}^{2}(\Omega)^{3}$ and $p \in W_{r}^{1}(\Omega)$, for some $r>3$, one needs additional geometrical restrictions on $\Omega$. More precisely, the dihedral angles must be less than $3 \pi / 4$ (cf. [21, sec. 5.5]). In [13, the authors argued that such a condition on $\Omega$ is essentially consistent with $(\vec{u}, p) \in W_{\infty}^{1}(\Omega)^{3} \times L^{\infty}(\Omega)$. The condition $\vec{u} \in W_{r}^{2}(\Omega)^{3}$, for some $r>3$ does imply by Sobolev embedding theorem $\vec{u} \in W_{\infty}^{1}(\Omega)^{3}$. However, for a general convex polyhedral domain $(\vec{u}, p)$ might not belong to $W_{r}^{2}(\Omega)^{3} \times W_{r}^{1}(\Omega)$ for any

Received by the editor May 21, 2010 and, in revised form, May 10, 2011 and August 18, 2011. 2010 Mathematics Subject Classification. Primary 65N30, 65N15.

Key words and phrases. Maximum norm, finite element, optimal error estimates, Stokes.

The first author was partially supported by NSF grant DMS-0914596.

The second author was partially supported by NSF grant DMS-0811167. 
$r>3$, but nevertheless Maz'ya and Rossmann [25] showed that $(\vec{u}, p)$ will always belong to $C^{1+\sigma}(\Omega)^{3} \times C^{\sigma}(\Omega)$, where the Hölder exponent $\sigma$ depends on $\Omega$. We use this important result to establish (1.2) for general convex polyhedral domains $\Omega$.

A standard argument applied to the above stability result gives the best approximation property:

$$
\begin{aligned}
& \left\|\nabla\left(\vec{u}-\vec{u}_{h}\right)\right\|_{L^{\infty}(\Omega)}+\left\|p-p_{h}\right\|_{L^{\infty}(\Omega)} \\
& \quad \leq C \min _{(\vec{\chi}, w) \in \vec{V}_{h} \times M_{h}}\left(\|\nabla(\vec{u}-\vec{\chi})\|_{L^{\infty}(\Omega)}+\|p-w\|_{L^{\infty}(\Omega)}\right) .
\end{aligned}
$$

Such estimates have many applications. In addition to the ones mentioned in [13, we would like to mention state-constrained optimal control problems [5]. In such problems the Lagrange multipliers are just measures and the pointwise stability estimates are essential.

Our proof is based on the technique developed in the series of papers by Schatz and Wahlbin (e.g. [31, 32, 33]) and is different from the global weighted technique used in [9, 13]. Our argument uses dyadic decomposition of $\Omega$ and requires local energy estimates together with sharp pointwise estimates for the corresponding components of the Green's matrix. For smooth domains such a technique was successfully used in [3] for mixed methods and [16] for discontinuous Galerkin (DG) methods, where higher-order regularity results were used. In the present paper we only assume $C^{1+\sigma}(\Omega)^{3} \times C^{\sigma}(\Omega)$ regularity. In order to prove (1.2) for $(\vec{u}, p) \in C^{1+\sigma}(\Omega)^{3} \times C^{\sigma}(\Omega)$ we need to develop several new tools. The first necessary ingredient is the new local energy estimates. Such estimates are important and have independent interest. They show how the error depends locally on the solution. Arnold and Liu [1 proved such estimates for subdomains away from the boundary. Later, those estimates were used in [3] to show (1.3) on smooth domains. In [16] such local energy estimates were extended up to the boundary for DG methods. The common feature of those estimates is the presence of the discrete pressure error term in some negative-order norm. Then by a duality argument the pressure term in weaker norm can be handled separately. However, such duality arguments require additional smoothness of the solution, which for general convex polyhedral domains do not hold. As a result, we cannot use those results directly. One of the main contributions of this paper is deriving new local energy estimates that do not involve the pressure error term (cf. Sec. 31). The second necessary ingredient is applying, in a careful way, sharp Hölder pointwise estimates for the components of the Green's matrix which were recently derived by Rossmann [28, (see also [18, 25] for similar results).

We would like to mention that similar Hölder type Green's function estimates were obtained in [17 for the Laplace equation and allowed the authors to obtain uniform stability of the Ritz projection for the Laplace equation on a general convex polyhedral domain. This paper can be considered as an extension of [17] to the Stokes problem (1.1). However, the Stokes problem is technically more challenging and involved. The main difficulty comes from the presence of the pressure term and the new local energy estimates for the Stokes problem will play a key role to overcome this difficulty.

The rest of the paper is organized as follows. In Section 2 we list the finite element assumptions and state the main result. Important analytical tools, local energy estimates and the pointwise estimates for the Green's matrix of the continuous problem are given in Sections 3 and 4 , respectively. In Section 5, we provide a 
proof of the main result. For technical reasons we first establish the stability for the velocity and then for the pressure. Finally, in Section 6 we comment on possible extensions and open problems.

\section{Assumptions and the Main Result}

Before stating the main result we list our assumptions on the finite element spaces.

2.1. Finite element approximation. For the finite element approximation of the problem, let $\mathcal{T}_{h}, 0<h<1$, be a sequence of partitions of $\Omega, \bar{\Omega}=\bigcup_{T \in \mathcal{T}_{h}} \bar{T}$, with the elements $T$ mutually disjoint. Let $h_{T}$ denote the diameter of $T$ and $h=\max _{T} h_{T}$. The partitions are face-to-face so that simplices meet only in full lower-dimensional faces or not at all. The simplices are assumed to be quasi-uniform, i.e. (if necessary after a renormalization of $h$ ), there exists a constant $C$ independent of $h$, such that

$$
\operatorname{diam} T \leq h_{T} \leq C(\text { meas } T)^{1 / 3}, \quad \forall T \in \mathcal{T}_{h} .
$$

The finite element velocity space is denoted by $\vec{V}_{h} \subset H_{0}^{1}(\Omega)^{3}$ and the pressure space is denoted by $M_{h} \subset L^{2}(\Omega)$. We assume that $\vec{V}_{h}$ contains the space of piecewise polynomials of degree $k$ and is contained in the space of piecewise polynomials of degree $l$. We assume that $M_{h}$ contains the space of polynomials of degree $k-1$.

The finite element approximation $\left(\vec{u}_{h}, p_{h}\right) \in\left(\vec{V}_{h} \times M_{h}\right)$ solves

$$
\begin{aligned}
\left(\nabla \vec{u}_{h}, \nabla \vec{v}\right)-\left(p_{h}, \nabla \cdot \vec{v}\right) & =(\vec{f}, \vec{v}), \quad \forall \vec{v} \in \vec{V}_{h}, \\
\left(q, \nabla \cdot \vec{u}_{h}\right) & =0, \quad \forall q \in M_{h},
\end{aligned}
$$

where $(\cdot, \cdot)$ denotes the usual $L^{2}(\Omega)$ inner product. The approximation to the pressure $p_{h}$ is unique up to a constant. We can for example require $p, p_{h} \in L_{0}^{2}$, i.e., $\int_{\Omega} p(x) d x=\int_{\Omega} p_{h}(x) d x=0$. Instead, we will require

$$
\int_{\Omega} p(x) \phi(x) d x=\int_{\Omega} p_{h}(x) \phi(x) d x=0,
$$

where $\phi(x)$ is an infinitely differentiable function on $\Omega$ that vanishes in a neighborhood of the edges and satisfies

$$
\int_{\Omega} \phi(x) d x=1
$$

Without loss of generality, we fix $\phi$ as above and assume $p, p_{h}$ satisfy (2.2). In other words, we let $p$ and $p_{h}$ belong to the space

$$
L_{\phi}^{2}(\Omega):=\left\{v \in L^{2}(\Omega): \int_{\Omega} v(x) \phi(x) d x=0\right\} .
$$

2.2. Assumptions. In the analysis below in order to establish the main result, we assume the existence of two projection operators $\mathbf{P}: H_{0}^{1}(\Omega)^{3} \rightarrow \vec{V}_{h}$ and $\mathbf{R}$ : $L^{2}(\Omega) \rightarrow M_{h}$ with the following properties:

Assumption 1 (Stability). There exists a constant $C$ independent of $h$ such that

$$
\|\mathbf{P} \vec{v}\|_{H^{1}(\Omega)} \leq C\|\vec{v}\|_{H^{1}(\Omega)}, \quad \forall \vec{v} \in H_{0}^{1}(\Omega)^{3} .
$$

Assumption 2 (Preservation of divergence).

$$
\left(\nabla \cdot(\vec{v}-\mathbf{P} \vec{v}), q_{h}\right)=0, \quad \forall q_{h} \in M_{h}, \quad \forall \vec{v} \in H_{0}^{1}(\Omega)^{3} .
$$


Assumption 3 (Approximation). Let $Q \subset Q_{d} \subset \Omega$, with $d \geq \kappa h$, for some fixed $\kappa$ sufficiently large and $Q_{d}=\{x \in \Omega: \operatorname{dist}(x, Q) \leq d\}$. For any $\vec{v} \in H^{\ell}\left(Q_{d}\right)^{3}$ there exists $C$ independent of $h$ and $\vec{v}$ such that

$$
\|\vec{v}-\mathbf{P} \vec{v}\|_{L^{2}(Q)}+h\|\vec{v}-\mathbf{P} \vec{v}\|_{H^{1}(Q)} \leq C h^{\ell}\|\vec{v}\|_{H^{\ell}\left(Q_{d}\right)} \quad \text { for } \ell=1,2 .
$$

For any $\vec{v} \in C^{1+\sigma}\left(Q_{d}\right)^{3}$ there exists $C$ independent of $h$ such that

$$
\|\vec{v}-\mathbf{P} \vec{v}\|_{W_{\infty}^{t}(Q)} \leq C h^{1+\sigma-t}\|\vec{v}\|_{C^{1+\sigma}\left(Q_{d}\right)}, \quad t=0,1,
$$

where

$$
\|\vec{v}\|_{C^{1+\sigma}(Q)}=\|\vec{v}\|_{C^{1}(Q)}+\sup _{\substack{x, y \in Q \\ i \in\{1,2,3\}}} \frac{\left|\overrightarrow{e_{i}} \cdot(\nabla \vec{v}(x)-\nabla \vec{v}(y))\right|}{|x-y|^{\sigma}} .
$$

Similar approximation properties we need for R. For any $q \in H^{1}\left(Q_{d}\right)$ there exists $C$ independent of $h$ and $q$ such that

$$
\|q-\mathbf{R} q\|_{L^{2}(Q)} \leq C h\|q\|_{H^{1}\left(Q_{d}\right)} .
$$

For any $q \in C^{\sigma}\left(Q_{d}\right)$ there exists $C$ independent of $h$ such that

$$
\|q-\mathbf{R} q\|_{L^{\infty}(Q)} \leq C h^{\sigma}\|q\|_{C^{\sigma}\left(Q_{d}\right)} .
$$

Assumption 4 (Superapproximation). Let $\omega \in C_{0}^{\infty}\left(Q_{d}\right)$ be a smooth cut-off function such that $\omega \equiv 1$ on $Q$ and

$$
\left|D^{s} \omega\right| \leq C d^{-s}, \quad s=0,1 .
$$

We assume,

$$
\left\|\nabla\left(\omega^{2} \vec{v}-\mathbf{P}\left(\omega^{2} \vec{v}\right)\right)\right\|_{L^{2}(Q)} \leq C d^{-1}\|\vec{v}\|_{L^{2}\left(Q_{d}\right)}, \quad \forall \vec{v} \in \vec{V}_{h}
$$

and

$$
\left\|\omega^{2} q-\mathbf{R}\left(\omega^{2} q\right)\right\|_{L^{2}(Q)} \leq C h d^{-1}\|q\|_{L^{2}\left(Q_{d}\right)}, \quad \forall q \in M_{h} .
$$

Assumption 5 (Inverse inequality). There is a constant $C$ independent of $h$ such that

$$
\|\vec{v}\|_{H^{1}(Q)} \leq C h^{-1}\|\vec{v}\|_{L^{2}\left(Q_{d}\right)}, \quad \forall \vec{v} \in \vec{V}_{h},
$$

In the proof of our pointwise estimates we will use the following energy error estimates.

Proposition 2.1. Let $(\vec{u}, p)$ solve (1.1) and $\left(\vec{u}_{h}, p_{h}\right)$ solve (2.1). Assume the above assumptions are satisfied, then there exists a constant $C$ independent of $h$ such that,

$$
\left\|\vec{u}-\vec{u}_{h}\right\|_{H^{1}(\Omega)}+\left\|p-p_{h}\right\|_{L^{2}(\Omega)} \leq C \min _{(\vec{\chi}, w) \in \vec{V}_{h} \times M_{h}}\left(\|\vec{u}-\vec{\chi}\|_{H^{1}(\Omega)}+\|p-w\|_{L^{2}(\Omega)}\right) .
$$

Remark 1. In some textbooks, (cf. [10, Prop. 4.14]), the proof assumes that $p \in L_{0}^{2}$, however, essentially the same proof holds for $p \in L_{\phi}^{2}$.

2.3. Examples of the subspaces. Several common finite element spaces for the Stokes problem are known to satisfy the above assumptions; for example, MINI and Taylor-Hood elements of degree greater than or equal to three. Operators satisfying Assumptions 2 25 were constructed in [13] and [14. For low-order MINI elements one can verify the assumptions by following the ideas in [1, Sec. 3]. 
2.4. Main Result. The main result establishes the stability of the gradient of the finite element velocity solution and the pressure in the $L^{\infty}$ norm.

Theorem 1. Let $(\vec{u}, p)$ and $\left(\vec{u}_{h}, p_{h}\right)$ satisfy (1.1) and (2.1), respectively. If the assumptions of Section 2.2 are met, then there exists a constant $C$ independent of $h$ such that

$$
\left\|\nabla \vec{u}_{h}\right\|_{L^{\infty}(\Omega)}+\left\|p_{h}\right\|_{L^{\infty}(\Omega)} \leq C\left(\|\nabla \vec{u}\|_{L^{\infty}(\Omega)}+\|p\|_{L^{\infty}(\Omega)}\right) .
$$

By a standard argument we have the following best approximation property.

Corollary 1. Under the assumptions of Theorem 1, there exists a constant $C$ independent of $h$ such that

$\left\|\nabla\left(\vec{u}-\vec{u}_{h}\right)\right\|_{L^{\infty}(\Omega)}+\left\|p-p_{h}\right\|_{L^{\infty}(\Omega)} \leq C \inf _{(\vec{\chi}, w) \in \vec{V}_{h} \times M_{h}}\left(\|\nabla(\vec{u}-\vec{\chi})\|_{L^{\infty}(\Omega)}+\|p-w\|_{L^{\infty}(\Omega)}\right)$.

Proof. The proof of the corollary follows easily by taking $\vec{u}-\vec{\chi}$ and $p-w$ with arbitrary $\vec{\chi} \in \vec{V}_{h}$ and $w \in M_{h}$ instead of $\vec{u}$ and $p$ in the stability estimate of Theorem 1 and using that the Stokes projection $\left(\vec{u}_{h}, p_{h}\right)$ is invariant on $\vec{V}_{h} \times M_{h}$.

\section{LOCAL ENERGy ESTIMATES}

Local energy estimates are essential to our proof. These estimates are important, although technical, and show how the error depends locally on the solution. Such estimates take their origin from the Caccioppoli inequality for the continuous problem. Consider two concentric balls $B_{1}$ and $B_{2}$ of radii $d$ and $2 d$, respectively, such that $B_{1} \Subset B_{2} \Subset \Omega$. In the interior of the domain, the Caccioppoli inequality says that if $\vec{v}$ is the solution of (1.1) with $\vec{f} \equiv \overrightarrow{0}$ on $B_{2}$, then there exists a constant $C$ independent of $\vec{v}$ and $d$ such that (cf. [12, Thm. 1.1])

$$
\|\nabla \vec{v}\|_{L^{2}\left(B_{1}\right)} \leq \frac{C}{d}\|\vec{v}\|_{L^{2}\left(B_{2}\right)} .
$$

In the finite element setting such estimates are not known and the pressure term usually in a weaker norm enters the estimates. First such interior local error estimates were derived in [1, Lem. 5.1] on subdomains away from the boundary. More precisely, they state that for any functions $\vec{v}_{h} \in \vec{V}_{h}$ and $q_{h} \in M_{h}$ satisfying

$$
\begin{aligned}
\left(\nabla \vec{v}_{h}, \nabla \vec{\chi}\right)+\left(q_{h}, \nabla \cdot \vec{\chi}\right) & =(f, \vec{\chi}), \quad \forall \vec{\chi} \in \vec{V}_{h}, \\
\left(\nabla \cdot \vec{v}_{h}, w\right) & =0, \quad \forall w \in M_{h},
\end{aligned}
$$

with $\vec{f} \equiv \overrightarrow{0}$ on $B_{2}$ and concentric balls $B_{1}$ and $B_{2}$ as above, and any nonnegative integer $t$,

$$
\begin{aligned}
\left\|\nabla \vec{v}_{h}\right\|_{L^{2}\left(B_{1}\right)}+\left\|q_{h}\right\|_{L^{2}\left(B_{1}\right)} & \leq C h d^{-1}\left(\left\|\nabla \vec{v}_{h}\right\|_{L^{2}\left(B_{2}\right)}+\left\|q_{h}\right\|_{L^{2}\left(B_{2}\right)}\right) \\
& +C d^{-t-1}\left(\left\|\vec{v}_{h}\right\|_{H^{-t}\left(B_{2}\right)}+\left\|q_{h}\right\|_{H^{-t-1}\left(B_{2}\right)}\right) .
\end{aligned}
$$

By a covering argument (cf. [27, Thm. 5.1]) the above estimate can be extended to any subdomains $A_{1} \Subset A_{2} \Subset \Omega$, with $d=\operatorname{dist}\left(\bar{A}_{1}, \partial A_{2}\right) \geq \kappa h$ for some fixed sufficiently large constant $\kappa$, with the assumption $\vec{f} \equiv \overrightarrow{0}$ on $A_{2}$. Using (3.2) the authors in 1 derived that the error satisfies,

$$
\begin{aligned}
\left\|\nabla\left(\vec{v}-\vec{v}_{h}\right)\right\|_{L^{2}\left(A_{1}\right)} & +\left\|q-q_{h}\right\|_{L^{2}\left(A_{1}\right)} \leq C\left(h^{r-1}\left(\|\vec{v}\|_{H^{r}\left(A_{2}\right)}+h\|q\|_{H^{r}\left(A_{2}\right)}\right)\right. \\
& \left.+d^{-t-1}\left\|\vec{v}-\vec{v}_{h}\right\|_{H^{-t}\left(A_{2}\right)}+d^{-t-1}\left\|q-q_{h}\right\|_{H^{-t-1}\left(A_{2}\right)}\right) .
\end{aligned}
$$


For details we refer to [1]. Similar local energy estimates were derived in [16] for discontinuous Galerkin methods for the Stokes problem on smooth domains.

The main common feature of the local error estimates in [1] and [16] is that they contain the pressure term in some negative norm on the right-hand side even if only the velocity error is to be estimated. Such results are not sufficient in our setting since handling such terms requires additional smoothness of the exact solution, which for general polyhedral domains we do not have. One significant contribution of our local energy estimates is that we avoid the pressure term in the negative norm at the expense of adding the original term on a slightly bigger subdomain multiplied by an arbitrary small number.

In order to state our result we consider $(\vec{v}, q) \in H_{0}^{1}(\Omega)^{3} \times L^{2}(\Omega)$ and $\left(\vec{v}_{h}, q_{h}\right) \in$ $\vec{V}_{h} \times M_{h}$ that satisfy the following orthogonality relation:

$$
\begin{aligned}
\left(\nabla\left(\vec{v}-\vec{v}_{h}\right), \nabla \vec{\chi}\right)+\left(q-q_{h}, \nabla \cdot \vec{\chi}\right) & =0, \quad \forall \vec{\chi} \in \vec{V}_{h}, \\
\left(\nabla \cdot\left(\vec{v}-\vec{v}_{h}\right), w\right) & =0, \quad \forall w \in M_{h} .
\end{aligned}
$$

Theorem 2. Suppose $(\vec{v}, q) \in H_{0}^{1}(\Omega)^{3} \times L^{2}(\Omega)$ and $\left(\vec{v}_{h}, q_{h}\right) \in \vec{V}_{h} \times M_{h}$ satisfy (3.3). Then, there exists a constant $C$ such that for every pair of sets $A_{1} \subset A_{2} \subset \Omega$ such that $\operatorname{dist}\left(\bar{A}_{1}, \partial A_{2} \backslash \partial \Omega\right) \geq d \geq \kappa h$ (for some fixed constant $\kappa$ sufficiently large) the following bound holds:

$$
\begin{aligned}
\left\|\nabla\left(\vec{v}-\vec{v}_{h}\right)\right\|_{L^{2}\left(A_{1}\right)} \leq & C\left(\|\nabla(\vec{v}-\mathbf{P} \vec{v})\|_{L^{2}\left(A_{2}\right)}+\|q-\mathbf{R} q\|_{L^{2}\left(A_{2}\right)}+\frac{1}{\varepsilon d}\|\vec{v}-\mathbf{P} \vec{v}\|_{L^{2}\left(A_{2}\right)}\right) \\
& +\varepsilon\left\|\nabla\left(\vec{v}-\vec{v}_{h}\right)\right\|_{L^{2}\left(A_{2}\right)}+\frac{C}{\varepsilon d}\left\|\vec{v}-\vec{v}_{h}\right\|_{L^{2}\left(A_{2}\right)} .
\end{aligned}
$$

The first three terms are usually referred to as approximation terms and the last two are the pollution terms. Notice, that there is no pollution of the pressure term in our estimates.

By a covering argument we will reduce the above result to the case $A_{s}=B_{s} \cap \Omega$ for $s>0$, where $B_{s}=B_{s d}\left(x_{0}\right)$ is a ball of radius $s d$ centered at $x_{0} \in \bar{\Omega}$. In the following analysis we will always assume $d \geq \kappa h$, for some $\kappa$ sufficiently large to allow several mesh layers. Before proving Theorem 2, first we establish the following lemma.

Lemma 3.1. Assume that there exists a ball $B \subset A_{1}$, such that $\operatorname{diam}\left(A_{1}\right) \leq d<$ $\rho \operatorname{diam}(B)$, where $\rho$ is a fixed constant that only depends on $\Omega$. Then, there exists a constant $C$ independent of $A_{1}, A_{2}, d$, and $h$ such that for any $0<\varepsilon<1$,

$$
\begin{aligned}
\left\|\nabla\left(\vec{v}-\vec{v}_{h}\right)\right\|_{L^{2}\left(A_{1}\right)} \leq & C\left(\|\nabla(\vec{v}-\mathbf{P} \vec{v})\|_{L^{2}\left(A_{2}\right)}+\|q-\mathbf{R} q\|_{L^{2}\left(A_{2}\right)}+\frac{C}{\varepsilon d}\|\vec{v}-\mathbf{P} \vec{v}\|_{L^{2}\left(A_{2}\right)}\right) \\
& +\varepsilon\left\|\nabla\left(\vec{v}-\vec{v}_{h}\right)\right\|_{L^{2}\left(A_{2}\right)}+\frac{C}{\varepsilon d}\left\|\vec{v}-\vec{v}_{h}\right\|_{L^{2}\left(A_{2}\right)} .
\end{aligned}
$$

Proof. Let $\omega \in C_{0}^{\infty}\left(A_{3 / 2}\right)$, be the cut-off function from Assumption 4 such that $\omega \equiv 1$ on $A_{1}$. Using the product rule

$$
\begin{aligned}
\left\|\nabla\left(\vec{v}-\vec{v}_{h}\right)\right\|_{L^{2}\left(A_{1}\right)}^{2} & \leq\left\|\omega \nabla\left(\vec{v}-\vec{v}_{h}\right)\right\|_{L^{2}(\Omega)}^{2}=\left(\nabla\left(\vec{v}-\vec{v}_{h}\right), \omega^{2} \nabla\left(\vec{v}-\vec{v}_{h}\right)\right) \\
& =\left(\nabla\left(\vec{v}-\vec{v}_{h}\right), \nabla\left(\omega^{2}\left(\vec{v}-\vec{v}_{h}\right)\right)\right)-\left(\nabla\left(\vec{v}-\vec{v}_{h}\right), \nabla\left(\omega^{2}\right) \otimes\left(\vec{v}-\vec{v}_{h}\right)\right),
\end{aligned}
$$

where $\vec{u} \otimes \vec{v}$ denotes a matrix with components $u_{i} v_{j}$ for $i, j=1,2,3$. 
By the Cauchy-Schwarz inequality and the property (2.6a), we get

$$
-\left(\nabla\left(\vec{v}-\vec{v}_{h}\right), \nabla\left(\omega^{2}\right) \otimes\left(\vec{v}-\vec{v}_{h}\right)\right) \leq \frac{C}{d}\left\|\omega \nabla\left(\vec{v}-\vec{v}_{h}\right)\right\|_{L^{2}(\Omega)}\left\|\vec{v}-\vec{v}_{h}\right\|_{L^{2}\left(A_{3 / 2}\right)} .
$$

By the arithmetic-geometric mean inequality and (3.4), we obtain

$$
\frac{1}{2}\left\|\omega \nabla\left(\vec{v}-\vec{v}_{h}\right)\right\|_{L^{2}(\Omega)}^{2} \leq\left(\nabla\left(\vec{v}-\vec{v}_{h}\right), \nabla\left(\omega^{2}\left(\vec{v}-\vec{v}_{h}\right)\right)\right)+\frac{C}{d^{2}}\left\|\vec{v}-\vec{v}_{h}\right\|_{L^{2}\left(A_{3 / 2}\right)}^{2} .
$$

Adding and subtracting $\mathbf{P} \vec{v}$ we obtain,

$$
\begin{aligned}
\left(\nabla\left(\vec{v}-\vec{v}_{h}\right), \nabla\left(\omega^{2}\left(\vec{v}-\vec{v}_{h}\right)\right)\right)= & \left(\nabla\left(\vec{v}-\vec{v}_{h}\right), \nabla\left(\omega^{2}\left(\mathbf{P} \vec{v}-\vec{v}_{h}\right)\right)\right) \\
& +\left(\nabla\left(\vec{v}-\vec{v}_{h}\right), \nabla\left(\omega^{2}(\vec{v}-\mathbf{P} \vec{v})\right)\right) .
\end{aligned}
$$

The second term on the right-hand side can be estimated as

$$
\begin{aligned}
& \left(\nabla\left(\vec{v}-\vec{v}_{h}\right), \nabla\left(\omega^{2}(\vec{v}-\mathbf{P} \vec{v})\right)\right) \\
& \quad \leq C\left\|\omega \nabla\left(\vec{v}-\vec{v}_{h}\right)\right\|_{L^{2}(\Omega)}\left(\|\nabla(\vec{v}-\mathbf{P} \vec{v})\|_{L^{2}\left(A_{3 / 2}\right)}+\frac{1}{d}\|\vec{v}-\mathbf{P} \vec{v}\|_{L^{2}\left(A_{3 / 2}\right)}\right),
\end{aligned}
$$

where we used (2.6a). Therefore, by (3.5),

$$
\begin{aligned}
\frac{1}{4}\left\|\omega \nabla\left(\vec{v}-\vec{v}_{h}\right)\right\|_{L^{2}(\Omega)}^{2} & \leq\left(\nabla\left(\vec{v}-\vec{v}_{h}\right), \nabla\left(\omega^{2}\left(\mathbf{P} \vec{v}-\vec{v}_{h}\right)\right)\right)+C\|\nabla(\vec{v}-\mathbf{P} \vec{v})\|_{L^{2}\left(A_{3 / 2}\right)}^{2} \\
& +\frac{C}{d^{2}}\|\vec{v}-\mathbf{P} \vec{v}\|_{L^{2}\left(A_{3 / 2}\right)}^{2}+\frac{C}{d^{2}}\left\|\vec{v}-\vec{v}_{h}\right\|_{L^{2}\left(A_{3 / 2}\right)}^{2} .
\end{aligned}
$$

Put

$$
\vec{\Psi}:=\omega^{2}\left(\mathbf{P} \vec{v}-\vec{v}_{h}\right) .
$$

Adding and subtracting $\nabla \mathbf{P} \vec{\Psi}$, we have

$$
\begin{aligned}
&\left(\nabla\left(\vec{v}-\vec{v}_{h}\right), \nabla\left(\omega^{2}\left(\mathbf{P} \vec{v}-\vec{v}_{h}\right)\right)\right)=\left(\nabla\left(\vec{v}-\vec{v}_{h}\right), \nabla \vec{\Psi}\right) \\
&=\left(\nabla\left(\vec{v}-\vec{v}_{h}\right), \nabla \mathbf{P} \vec{\Psi}\right)+\left(\nabla\left(\vec{v}-\vec{v}_{h}\right), \nabla(\vec{\Psi}-\mathbf{P} \vec{\Psi})\right):=I_{1}+I_{2} .
\end{aligned}
$$

Hence in view of (3.6),

$$
\begin{aligned}
\frac{1}{4}\left\|\omega \nabla\left(\vec{v}-\vec{v}_{h}\right)\right\|_{L^{2}(\Omega)}^{2} \leq & I_{1}+I_{2}+C\|\nabla(\vec{v}-\mathbf{P} \vec{v})\|_{L^{2}\left(A_{3 / 2}\right)}^{2} \\
& +\frac{C}{d^{2}}\|\vec{v}-\mathbf{P} \vec{v}\|_{L^{2}\left(A_{3 / 2}\right)}^{2}+\frac{C}{d^{2}}\left\|\vec{v}-\vec{v}_{h}\right\|_{L^{2}\left(A_{3 / 2}\right)}^{2} .
\end{aligned}
$$

To estimate $I_{2}$ we apply the Cauchy-Schwarz inequality and the superapproximation Assumption 4, and the arithmetic-geometric mean inequality to obtain,

$$
\begin{aligned}
I_{2} & \leq\left\|\nabla\left(\vec{v}-\vec{v}_{h}\right)\right\|_{L^{2}\left(A_{3 / 2}\right)}\|\nabla(\vec{\Psi}-\mathbf{P} \vec{\Psi})\|_{L^{2}\left(A_{3 / 2}\right)} \\
& \leq\left\|\nabla\left(\vec{v}-\vec{v}_{h}\right)\right\|_{L^{2}\left(A_{3 / 2}\right)} \frac{C}{d}\left\|\mathbf{P} \vec{v}-\vec{v}_{h}\right\|_{L^{2}\left(A_{2}\right)} \\
& \leq \varepsilon\left\|\nabla\left(\vec{v}-\vec{v}_{h}\right)\right\|_{L^{2}\left(A_{3 / 2}\right)}^{2}+\frac{C}{\varepsilon d^{2}}\left(\|\mathbf{P} \vec{v}-\vec{v}\|_{L^{2}\left(A_{2}\right)}^{2}+\left\|\vec{v}-\vec{v}_{h}\right\|_{L^{2}\left(A_{2}\right)}^{2}\right),
\end{aligned}
$$

for any $0<\varepsilon<1$. To estimate $I_{1}$ we use (3.3a), then add and subtract $\nabla \cdot \vec{\Psi}$ and use the property of $\mathbf{P}$ from Assumption 2, to obtain

$$
I_{1}=-\left(q-q_{h}, \nabla \cdot \mathbf{P} \vec{\Psi}\right)=-\left(q-q_{h}, \nabla \cdot \vec{\Psi}\right)-(q-\mathbf{R} q, \nabla \cdot(\mathbf{P} \vec{\Psi}-\vec{\Psi})):=I_{3}+I_{4}
$$


Next we estimate $I_{4}$. Similar to the estimate for $I_{2}$ we use the superapproximation property (2.6b) and the arithmetic-geometric mean inequality to obtain,

$$
\begin{aligned}
I_{4} & \leq\|q-\mathbf{R} q\|_{L^{2}\left(A_{3 / 2}\right)}\|\nabla \cdot(\mathbf{P} \vec{\Psi}-\vec{\Psi})\|_{L^{2}\left(A_{3 / 2}\right)} \\
& \leq\|q-\mathbf{R} q\|_{L^{2}\left(A_{3 / 2}\right)} \frac{C}{d}\left\|\mathbf{P} v-v_{h}\right\|_{L^{2}\left(A_{2}\right)} \\
& \leq\|q-\mathbf{R} q\|_{L^{2}\left(A_{3 / 2}\right)}^{2}+\frac{C}{d^{2}}\left(\left\|\vec{v}-\vec{v}_{h}\right\|_{L^{2}\left(A_{2}\right)}^{2}+\|\vec{v}-\mathbf{P} \vec{v}\|_{L^{2}\left(A_{2}\right)}^{2}\right) .
\end{aligned}
$$

Hence, combining these estimates we have

$$
\begin{aligned}
& \frac{1}{4}\left\|\omega \nabla\left(\vec{v}-\vec{v}_{h}\right)\right\|_{L^{2}(\Omega)}^{2} \leq I_{3}+\varepsilon\left\|\nabla\left(\vec{v}-\vec{v}_{h}\right)\right\|_{L^{2}\left(A_{3 / 2}\right)}^{2}+\|q-\mathbf{R} q\|_{L^{2}\left(A_{3 / 2}\right)}^{2} \\
& +C\|\nabla(\vec{v}-\mathbf{P} \vec{v})\|_{L^{2}\left(A_{2}\right)}^{2}+\frac{C}{\varepsilon d^{2}}\|\vec{v}-\mathbf{P} \vec{v}\|_{L^{2}\left(A_{2}\right)}^{2}+\frac{C}{\varepsilon d^{2}}\left\|\vec{v}-\vec{v}_{h}\right\|_{L^{2}\left(A_{2}\right)}^{2} .
\end{aligned}
$$

It remains to estimate $I_{3}$. Adding and subtracting $\mathbf{R} q$, we have

$$
I_{3}=-\left(q-q_{h}, \nabla \cdot \vec{\Psi}\right)=-\left(\mathbf{R} q-q_{h}, \nabla \cdot \vec{\Psi}\right)-(q-\mathbf{R} q, \nabla \cdot \vec{\Psi}):=I_{5}+I_{6} .
$$

We can estimate $I_{6}$ by using the Cauchy-Schwarz inequality, property (2.6a), and the arithmetic-geometric mean and triangle inequalities to obtain,

$$
\begin{aligned}
I_{6} \leq & C\|q-\mathbf{R} q\|_{L^{2}\left(A_{3 / 2}\right)}^{2}+\frac{1}{8}\left\|\omega \nabla\left(\vec{v}-\vec{v}_{h}\right)\right\|_{L^{2}\left(A_{3 / 2}\right)}^{2} \\
& +C\|\nabla(\vec{v}-\mathbf{P} \vec{v})\|_{L^{2}\left(A_{3 / 2}\right)}^{2}+\frac{C}{d^{2}}\|\vec{v}-\mathbf{P} \vec{v}\|_{L^{2}\left(A_{3 / 2}\right)}^{2}+\frac{C}{d^{2}}\left\|\vec{v}-\vec{v}_{h}\right\|_{L^{2}\left(A_{3 / 2}\right)}^{2},
\end{aligned}
$$

and hence,

$$
\begin{gathered}
\frac{1}{8}\left\|\omega \nabla\left(\vec{v}-\vec{v}_{h}\right)\right\|_{L^{2}(\Omega)}^{2} \leq I_{5}+\varepsilon\left\|\nabla\left(\vec{v}-\vec{v}_{h}\right)\right\|_{L^{2}\left(A_{3 / 2}\right)}^{2}+\|q-\mathbf{R} q\|_{L^{2}\left(A_{3 / 2}\right)}^{2} \\
\quad+C\|\nabla(\vec{v}-\mathbf{P} \vec{v})\|_{L^{2}\left(A_{2}\right)}^{2}+\frac{C}{\varepsilon d^{2}}\|\vec{v}-\mathbf{P} \vec{v}\|_{L^{2}\left(A_{2}\right)}^{2}+\frac{C}{\varepsilon d^{2}}\left\|\vec{v}-\vec{v}_{h}\right\|_{L^{2}\left(A_{2}\right)}^{2} .
\end{gathered}
$$

To estimate $I_{5}$ we note that $\vec{\Psi}$ vanishes on the boundary and as a result

$$
(c, \nabla \cdot \vec{\Psi})=0,
$$

for any constant $c$. Hence, for an arbitrary constant $c$ we have

$$
\begin{aligned}
I_{5} & =-\left(\mathbf{R} q-q_{h}-c, \nabla \cdot \vec{\Psi}\right)=-\left(\mathbf{R} q-q_{h}-c, \nabla \cdot \omega^{2}\left(\mathbf{P} \vec{v}-\vec{v}_{h}\right)\right) \\
& =-\left(\mathbf{R} q-q_{h}-c,\left(\nabla \omega^{2}\right) \cdot\left(\mathbf{P} \vec{v}-\vec{v}_{h}\right)\right)-\left(\mathbf{R} q-q_{h}-c, \omega^{2} \nabla \cdot\left(\mathbf{P} \vec{v}-\vec{v}_{h}\right)\right) .
\end{aligned}
$$

Setting

$$
\psi:=\mathbf{R} q-q_{h}-c
$$

and using that $\left(\nabla \cdot\left(\mathbf{P} \vec{v}-\vec{v}_{h}\right), \chi\right)=0$ for any $\chi \in M_{h}$, which follows from (3.3b) and (2.4), we have

$$
\begin{aligned}
I_{5} & =-\left(\psi,\left(\nabla \omega^{2}\right) \cdot\left(\mathbf{P} \vec{v}-\vec{v}_{h}\right)\right)-\left(\omega^{2} \psi, \nabla \cdot\left(\mathbf{P} \vec{v}-\vec{v}_{h}\right)\right) \\
& =-\left(\psi,\left(\nabla \omega^{2}\right) \cdot\left(\mathbf{P} \vec{v}-\vec{v}_{h}\right)\right)-\left(\omega^{2} \psi-\mathbf{R}\left(\omega^{2} \psi\right), \nabla \cdot\left(\mathbf{P} \vec{v}-\vec{v}_{h}\right)\right) .
\end{aligned}
$$

Using the superapproximation estimate (2.6c) and the inverse estimate (2.7a) we can bound the second term as follows:

$$
\begin{aligned}
\left(\omega^{2} \psi-\mathbf{R}\left(\omega^{2} \psi\right), \nabla \cdot\left(\mathbf{P} \vec{v}-\vec{v}_{h}\right)\right) & \leq \frac{C h}{d}\|\psi\|_{L^{2}\left(A_{3 / 2}\right)}\left\|\nabla\left(\mathbf{P} \vec{v}-\vec{v}_{h}\right)\right\|_{L^{2}\left(A_{3 / 2}\right)} \\
& \leq \frac{C}{d}\|\psi\|_{L^{2}\left(A_{3 / 2}\right)}\left\|\mathbf{P} \vec{v}-\vec{v}_{h}\right\|_{L^{2}\left(A_{2}\right)} .
\end{aligned}
$$


The first term is also bounded by the right-hand side above and as a result

$$
\begin{aligned}
I_{5} & \leq \frac{C}{d}\|\psi\|_{L^{2}\left(A_{3 / 2}\right)}\left\|\mathbf{P} \vec{v}-\vec{v}_{h}\right\|_{L^{2}\left(A_{2}\right)} \\
& \leq \varepsilon\|\psi\|_{L^{2}\left(A_{2}\right)}^{2}+\frac{C}{\varepsilon d^{2}}\left(\|\mathbf{P} \vec{v}-\vec{v}\|_{L^{2}\left(A_{2}\right)}^{2}+\left\|\vec{v}-\vec{v}_{h}\right\|_{L^{2}\left(A_{2}\right)}^{2}\right) .
\end{aligned}
$$

Combining, we get

$$
\begin{aligned}
\frac{1}{8}\left\|\omega \nabla\left(\vec{v}-\vec{v}_{h}\right)\right\|_{L^{2}(\Omega)}^{2} \leq & \varepsilon\|\psi\|_{L^{2}\left(A_{3 / 2}\right)}^{2}+\varepsilon\left\|\nabla\left(\vec{v}-\vec{v}_{h}\right)\right\|_{L^{2}\left(A_{2}\right)}^{2} \\
& +C\|q-\mathbf{R} q\|_{L^{2}\left(A_{2}\right)}^{2}+C\|\nabla(\vec{v}-\mathbf{P} \vec{v})\|_{L^{2}\left(A_{2}\right)}^{2} \\
& +\frac{C}{\varepsilon d^{2}}\|\vec{v}-\mathbf{P} \vec{v}\|_{L^{2}\left(A_{2}\right)}^{2}+\frac{C}{\varepsilon d^{2}}\left\|\vec{v}-\vec{v}_{h}\right\|_{L^{2}\left(A_{2}\right)}^{2} .
\end{aligned}
$$

We choose constant $c$ such that $\psi$ has zero mean on $A_{3 / 2}$. To estimate $\|\psi\|_{L^{2}\left(A_{3 / 2}\right)}$ we require the following lemma.

Lemma 3.2. Assume the hypothesis of Lemma 3.1 holds. Suppose the constant $c$ is such that $\psi=\mathbf{R} q-q_{h}-c$ has mean zero on $A_{3 / 2}$. Then, there exists a constant $C$ independent of $A_{3 / 2}$ and $\psi$, but that depends on $\rho$ (see Lemma 3.1) such that

$$
\|\psi\|_{L^{2}\left(A_{3 / 2}\right)} \leq C\left(\left\|\nabla\left(\vec{v}-\vec{v}_{h}\right)\right\|_{L^{2}\left(A_{2}\right)}+\|q-\mathbf{R} q\|_{L^{2}\left(A_{2}\right)}\right) .
$$

We postpone the proof of this result until the end of this section and finish the proof of Lemma 3.1. Using the above lemma we obtain,

$$
\begin{aligned}
& \frac{1}{8}\left\|\omega \nabla\left(\vec{v}-\vec{v}_{h}\right)\right\|_{L^{2}(\Omega)}^{2} \leq C \varepsilon\left\|\nabla\left(\vec{v}-\vec{v}_{h}\right)\right\|_{L^{2}\left(A_{2}\right)}^{2}+C\|q-\mathbf{R} q\|_{L^{2}\left(A_{2}\right)}^{2} \\
& \quad+C\|\nabla(\vec{v}-\mathbf{P} \vec{v})\|_{L^{2}\left(A_{2}\right)}^{2}+\frac{C}{\varepsilon d^{2}}\|\vec{v}-\mathbf{P} \vec{v}\|_{L^{2}\left(A_{2}\right)}^{2}+\frac{C}{\varepsilon d^{2}}\left\|\vec{v}-\vec{v}_{h}\right\|_{L^{2}\left(A_{2}\right)}^{2} .
\end{aligned}
$$

This completes the proof after re-defining $\varepsilon$.

\subsection{Proof of Lemma 3.2,}

Proof. Define $\vec{w} \in H_{0}^{1}\left(A_{3 / 2}\right)$ by

$$
\begin{aligned}
\nabla \cdot \vec{w}=\psi & \text { in } A_{3 / 2}, \\
\vec{w}=0 & \text { on } \partial A_{3 / 2} .
\end{aligned}
$$

We can choose $\vec{w}$ so that the following bound exists

$$
\|\vec{w}\|_{H^{1}\left(A_{3 / 2}\right)} \leq C\|\psi\|_{L^{2}\left(A_{3 / 2}\right)} .
$$

By Lemma 3.1 of Chapter III.3 in 11, the constant $C$ is independent of $\psi$ and depends only on the ratio of the diameter $A_{3 / 2}$ and the radius of the largest ball that can be inscribed into $A_{3 / 2}$ and hence by our hypothesis only depends on $\rho$. Let us extend $\vec{w}$ on all of $\Omega$ by zero outside of $A_{3 / 2}$. We note then that this implies that $\mathbf{P} \vec{w}$ vanishes outside of $A_{2}$ by (2.5a).

We have,

$$
\begin{aligned}
\|\psi\|_{L^{2}\left(A_{3 / 2}\right)}^{2} & =(\psi, \psi)_{A_{3 / 2}}=(\psi, \nabla \cdot \vec{w})_{A_{3 / 2}}=(\psi, \nabla \cdot \vec{w}) \\
& =\left(\mathbf{R} q-q_{h}-c, \nabla \cdot \vec{w}\right) \\
& =\left(\mathbf{R} q-q_{h}, \nabla \cdot \vec{w}\right) \\
& =\left(q-q_{h}, \nabla \cdot \vec{w}\right)+(\mathbf{R} q-q, \nabla \cdot \vec{w}) .
\end{aligned}
$$


Using (3.3a), (2.4), the Cauchy-Schwarz inequality and the stability of $\mathbf{P}$, we have

$$
\begin{aligned}
\left(q-q_{h},\right. & \nabla \cdot \vec{w})=\left(q-q_{h}, \nabla \cdot \mathbf{P} \vec{w}\right)+\left(q-q_{h}, \nabla \cdot(\vec{w}-\mathbf{P} \vec{w})\right) \\
& =\left(\nabla\left(\vec{v}-\vec{v}_{h}\right), \nabla \mathbf{P} \vec{w}\right)+(q-\mathbf{R} q, \nabla \cdot(\vec{w}-\mathbf{P} \vec{w})) \\
& \leq\left\|\nabla\left(\vec{v}-\vec{v}_{h}\right)\right\|_{L^{2}\left(A_{2}\right)}\|\nabla \mathbf{P} \vec{w}\|_{L^{2}\left(A_{2}\right)}+\|q-\mathbf{R} q\|_{L^{2}\left(A_{2}\right)}\|\nabla \cdot(\vec{w}-\mathbf{P} \vec{w})\|_{L^{2}\left(A_{2}\right)} \\
& \leq C\left(\left\|\nabla\left(\vec{v}-\vec{v}_{h}\right)\right\|_{L^{2}\left(A_{2}\right)}+\|q-\mathbf{R} q\|_{L^{2}\left(A_{2}\right)}\right)\|\vec{w}\|_{H^{1}\left(A_{3 / 2}\right)} .
\end{aligned}
$$

Hence, we get

$$
\begin{aligned}
\|\psi\|_{L^{2}\left(A_{3 / 2}\right)}^{2} & \leq C\left(\left\|\nabla\left(\vec{v}-\vec{v}_{h}\right)\right\|_{L^{2}\left(A_{2}\right)}+\|\mathbf{R} q-q\|_{L^{2}\left(A_{2}\right)}\right)\|\vec{w}\|_{H^{1}\left(A_{3 / 2}\right)} \\
& \leq C\left(\left\|\nabla\left(\vec{v}-\vec{v}_{h}\right)\right\|_{L^{2}\left(A_{2}\right)}+\|\mathbf{R} q-q\|_{L^{2}\left(A_{2}\right)}\right)\|\psi\|_{L^{2}\left(A_{3 / 2}\right)} .
\end{aligned}
$$

Therefore, dividing both sides by $\|\psi\|_{L^{2}\left(A_{3 / 2}\right)}$ we obtain the lemma.

\subsection{Proof of Theorem 2 ,}

Proof. Let $A_{1} \subset A_{2} \subset \Omega$, be such that $\operatorname{dist}\left(\bar{A}_{1}, \partial A_{2} \backslash \partial \Omega\right) \geq d \geq \kappa h$. It is not difficult to construct a covering $\left\{G_{i}\right\}_{i=1}^{M}$ of $A_{1}$, where $G_{i}=B_{\frac{d}{2}}\left(x_{i}\right) \cap \Omega$ with the following properties.

(1) $A_{1} \subset \bigcup_{i=1}^{M} G_{i}$.

(2) $x_{i} \in \bar{A}_{1}$ for each $1 \leq i \leq M$.

(3) Let $H_{i}=B_{d}\left(x_{i}\right) \cap \Omega$. There exists a fixed number $L$ such that each point $x \in \bigcup_{i=1}^{M} H_{i}$ is contained in at most $L$ sets from $\left\{H_{j}\right\}_{j=1}^{M}$.

(4) There exists a $\rho>0$ such that for each $1 \leq i \leq M$ there exists a ball $B \subset G_{i}$ such that $\operatorname{diam}\left(G_{i}\right) \leq \rho \operatorname{diam}(B)$.

Since $\operatorname{dist}\left(\bar{A}_{1}, \partial A_{2} \backslash \partial \Omega\right) \geq d$, using (2) we have that $\bigcup_{i=1}^{M} H_{i} \subset A_{2}$.

Applying Lemma 3.1 and using (1) and (4) we have

$$
\begin{aligned}
& \left\|\nabla\left(\vec{v}-\vec{v}_{h}\right)\right\|_{L^{2}\left(A_{1}\right)}^{2} \leq \sum_{i=1}^{M}\left\|\nabla\left(\vec{v}-\vec{v}_{h}\right)\right\|_{L^{2}\left(G_{i}\right)}^{2} \\
& \leq \sum_{i=1}^{M} C\left(\|\nabla(\vec{v}-\mathbf{P} \vec{v})\|_{L^{2}\left(H_{i}\right)}^{2}+\|q-\mathbf{R} q\|_{L^{2}\left(H_{i}\right)}^{2}+\left(\frac{1}{\varepsilon d}\right)^{2}\|\vec{v}-\mathbf{P} \vec{v}\|_{L^{2}\left(H_{i}\right)}^{2}\right. \\
& \left.\quad+\left(\frac{1}{\varepsilon d}\right)^{2}\left\|\vec{v}-\vec{v}_{h}\right\|_{L^{2}\left(H_{i}\right)}^{2}\right)+\varepsilon^{2}\left\|\nabla\left(\vec{v}-\vec{v}_{h}\right)\right\|_{L^{2}\left(H_{i}\right)}^{2} .
\end{aligned}
$$

Using (3) we have

$$
\begin{aligned}
& \left\|\nabla\left(\vec{v}-\vec{v}_{h}\right)\right\|_{L^{2}\left(A_{1}\right)}^{2} \leq C L\left(\|\nabla(\vec{v}-\mathbf{P} \vec{v})\|_{L^{2}\left(A_{2}\right)}^{2}+\|q-\mathbf{R} q\|_{L^{2}\left(A_{2}\right)}^{2}\right. \\
& \left.\quad+\left(\frac{1}{\varepsilon d}\right)^{2}\|\vec{v}-\mathbf{P} \vec{v}\|_{L^{2}\left(A_{2}\right)}^{2}+\left(\frac{1}{\varepsilon d}\right)^{2}\left\|\vec{v}-\vec{v}_{h}\right\|_{L^{2}\left(A_{2}\right)}^{2}\right)+L \varepsilon^{2}\left\|\nabla\left(\vec{v}-\vec{v}_{h}\right)\right\|_{L^{2}\left(A_{2}\right)}^{2} .
\end{aligned}
$$

This completes the proof after redefining $\varepsilon$.

\section{Maximum modulus estimates for the Green's matrix ON POLYHEDRAL TYPE DOMAINS}

The second important ingredient of our proof is the sharp pointwise Green's matrix estimates for the continuous problem, which we will introduce next. 
Let $\phi(x)$ be an infinitely differentiable function in $\Omega$ which vanishes in a neighborhood of the edges such that

$$
\int_{\Omega} \phi(x) d x=1
$$

The matrix

$$
G(x, \xi)=\left(G_{i, j}(x, \xi)\right)_{i, j=1}^{4},
$$

is called the Green's matrix for the problem (4.5) if the vector functions

$$
\vec{G}_{j}=\left(G_{1, j}, G_{2, j}, G_{3, j}\right)^{T}
$$

and the functions $G_{4, j}$ for $j=1,2,3,4$ are solutions of the problem

$$
\begin{aligned}
-\Delta_{x} \vec{G}_{j}(x, \xi)+\nabla_{x} G_{4, j}(x, \xi) & =\delta(x-\xi)\left(\delta_{1, j}, \delta_{2, j}, \delta_{3, j}\right)^{T}, \quad \text { for } x, \xi \in \Omega \\
-\nabla_{x} \cdot \vec{G}_{j}(x, \xi) & =(\delta(x-\xi)-\phi(x)) \delta_{4, j}, \quad \text { for } x, \xi \in \Omega \\
\vec{G}_{j}(x, \xi) & =\overrightarrow{0}, \quad \text { for } x \in \partial \Omega, \xi \in \Omega
\end{aligned}
$$

and $G_{4, j}$ satisfies the condition

$$
\int_{\Omega} G_{4, j}(x, \xi) \phi(x) d x=0, \quad \text { for } \quad \xi \in \Omega, \quad j=1,2,3,4 .
$$

Here, $\delta(x)$ is the delta function, and $\delta_{i, j}$ is the Kronecker delta symbol. In addition,

$$
G_{i, j}(x, \xi)=G_{j, i}(\xi, x) \quad \text { for } x, \xi \in \Omega, i, j=1,2,3,4 .
$$

The following theorem, (cf. [23, 24] and [20, Thm. 4.5]) gives us the existence and uniqueness of such a matrix.

Theorem 3. There exists a uniquely determined Green's matrix $G(x, \xi)$ such that the vector functions

$$
x \rightarrow \zeta(x, \xi)\left(\vec{G}_{j}(x, \xi), G_{4, j}(x, \xi)\right)
$$

belong to the space $H_{0}^{1}(\Omega)^{3} \times L^{2}(\Omega)$ for each $\xi \in \Omega$ and for every infinitely differentiable function $\zeta(\cdot, \xi)$ equal to zero in a neighborhood of the point $x=\xi$.

We will also need to consider the Stokes problem with non-zero divergence. Let $(\vec{u}, p) \in H_{0}^{1}(\Omega) \times L_{\phi}^{2}(\Omega)$ solve

$$
\begin{array}{rlrl}
-\Delta \vec{u}+\nabla p=\vec{f} & & \text { in } \Omega, \\
-\nabla \cdot \vec{u}=q & & \text { in } \Omega, \\
\vec{u} & =\overrightarrow{0} & & \text { on } \partial \Omega,
\end{array}
$$

for arbitrary $\vec{f} \in H^{-1}(\Omega)^{3}$ and $q \in L_{0}^{2}(\Omega)$ with $q$ vanishing on the singular points of $\Omega$; see [4. If $q \in H^{1}(\Omega) \cap L_{0}^{2}(\Omega)$ and vanishes on the edges of $\Omega$ and $\vec{f} \in L^{2}(\Omega)^{3}$ we have the following elliptic regularity result [4, 21,

$$
\|\vec{u}\|_{H^{2}(\Omega)}+\|p\|_{H^{1}(\Omega)} \leq C\left(\|\vec{f}\|_{L^{2}(\Omega)}+\|q\|_{H^{1}(\Omega)}\right) .
$$


Furthermore, the components of $(\vec{u}, p)$ admit the following representation (cf. 29]) in terms of the Green's matrix

$$
\begin{aligned}
u_{i}(x) & =\sum_{j=1}^{3} \int_{\Omega} G_{i, j}(x, \xi) f_{j}(\xi) d \xi+\int_{\Omega} G_{i, 4}(x, \xi) q(\xi) d \xi, \quad i=1,2,3 \\
p(x) & =\sum_{j=1}^{3} \int_{\Omega} G_{4, j}(x, \xi) f_{j}(\xi) d \xi+\int_{\Omega} G_{4,4}(x, \xi) q(\xi) d \xi .
\end{aligned}
$$

Next we state maximum modulus estimates for the Green's function in polyhedral domains. The first estimate was established in papers of 20, 23, 24, (see also 22, Sec. 11.5]). The second sharper estimate was established recently in [28.

Theorem 4. Let $\Omega \subset \mathbb{R}^{3}$ be a convex domain of polyhedral type. Then there exists a constant $C$ such that

$$
\begin{aligned}
& \left|\partial_{x}^{\alpha} \partial_{\xi}^{\beta} G_{i, j}(x, \xi)\right| \leq C|x-\xi|^{-1-|\alpha|-|\beta|-\delta_{i, 4}-\delta_{j, 4}}, \\
& \quad \text { for }|\alpha| \leq 1-\delta_{i, 4},|\beta| \leq 1-\delta_{j, 4},
\end{aligned}
$$

for $x, \xi \in \Omega, x \neq \xi$, and multi-indices $0 \leq|\alpha|,|\beta| \leq 1$.

Moreover, for polyhedral domain the Green's matrix satisfies the Hölder type estimate,

$$
\begin{aligned}
& \frac{\left|\partial_{x}^{\alpha} \partial_{\xi}^{\beta} G_{i, j}(x, \xi)-\partial_{y}^{\alpha} \partial_{\xi}^{\beta} G_{i, j}(y, \xi)\right|}{|x-y|^{\sigma}} \\
& \quad \leq C\left(|x-\xi|^{-1-\sigma-\delta_{j, 4}-\delta_{i, 4}-|\beta|-|\alpha|}+|y-\xi|^{-1-\sigma-\delta_{j, 4}-\delta_{i, 4}-|\beta|-|\alpha|}\right),
\end{aligned}
$$

for $|\alpha| \leq 1-\delta_{i, 4}$ and $|\beta| \leq 1-\delta_{j, 4}$. Here $\sigma$ is a sufficiently small positive number which depends on the geometry of the domain.

Here and in the rest of the paper we adopt the standard multi-index notation. Thus, for a multi-index $\alpha=\left(\alpha_{1}, \alpha_{2}, \alpha_{3}\right)$, we denote $|\alpha|=\alpha_{1}+\alpha_{2}+\alpha_{3}$ and $\partial_{x}^{\alpha}=$ $\frac{\partial^{|\alpha|}}{\partial x_{1}^{\alpha_{1}} \partial x_{2}^{\alpha_{2}} \partial x_{3}^{\alpha_{3}}}$.

\section{Proof of the MAIN ReSUlt}

For technical reasons we split the proof of our main result, Theorem 11, into two parts: stability of the gradient of the velocity and the stability of the pressure. First we will deal with the velocity.

5.1. Part 1, Velocity. Let $z$ be an arbitrary point of $\bar{\Omega}$ and let $T_{z} \in \mathcal{T}_{h}$ contain $z$. We will estimate $\left|\partial_{x_{j}}\left(\vec{u}_{h}\right)_{i}(z)\right|$, where $1 \leq i, j \leq 3$ are arbitrary. The idea of the proof is to represent the discrete solution in terms of the smooth Green's function. Then after some manipulations the problem is reduced to estimating the error of the Green's function in $L^{1}(\Omega)$ norm. To start we define a smooth delta function. Let $\delta_{h}^{z}(x)=\delta_{h} \in C_{0}^{1}\left(T_{z}\right)$ be a smooth function such that

$$
r(z)=\left(r, \delta_{h}\right)_{T_{z}}, \quad \forall r \in P^{l}\left(T_{z}\right),
$$

where $P^{l}\left(T_{z}\right)$ is the space of polynomials of degree at most $l$ defined on $T_{z}$, with the properties

$$
\left\|\delta_{h}\right\|_{W_{q}^{k}\left(T_{z}\right)} \leq C h^{-k-3(1-1 / q)}, \quad 1 \leq q \leq \infty, k=0,1 .
$$


Thus, in particular, $\left\|\delta_{h}\right\|_{L^{1}\left(T_{z}\right)} \leq C$ and $\left\|\delta_{h}\right\|_{L^{2}\left(T_{z}\right)} \leq C h^{-3 / 2}$. The explicit construction of a such function is given in [33, Appendix].

Next, we define the approximate Green's function $(\vec{g}, \lambda) \in H_{0}^{1}(\Omega)^{3} \times L_{\phi}^{2}(\Omega)$ to be the solution of the following equation:

$$
\begin{aligned}
-\triangle \vec{g}+\nabla \lambda & =\left(\partial_{x_{j}} \delta_{h}\right) \overrightarrow{e_{i}} \quad \text { in } \Omega, \\
\nabla \cdot \vec{g} & =0 \quad \text { in } \Omega, \\
\vec{g} & =\overrightarrow{0} \quad \text { on } \partial \Omega .
\end{aligned}
$$

Here $\overrightarrow{e_{i}}$ is the $i$-th standard basis vector in $\mathbb{R}^{3}$ and will be fixed throughout the paper. Again, $\lambda$ is unique up to a constant.

In the course of the proof we will need to estimate $\vec{g}$ and $\lambda$ in certain Hölder norms on subdomains away from the singular point $z$.

Lemma 5.1. Let $D \subset \Omega$ be such that $\operatorname{dist}(D, z) \geq d \geq 2 h$. Then there exists a constant $C$ independent of $d$ and $D$ such that

$$
\|\vec{g}\|_{C^{1+\sigma}(D)}+\|\lambda\|_{C^{\sigma}(D)} \leq C d^{-3-\sigma} .
$$

Proof. Using the Green's function representation 4.7a with $q=0$ and recalling that index $i$ in the definition of regularized Green's function $(\vec{g}, \lambda)$ in (5.3) is fixed, we have,

$$
\begin{aligned}
\partial_{x} g_{k}(x)-\partial_{y} g_{k}(y) & =\int_{\Omega}\left(\partial_{x} G_{k, i}(x, \xi)-\partial_{y} G_{k, i}(y, \xi)\right) \partial_{\xi}\left(\delta_{h}(\xi)\right) d \xi \\
& =-\int_{T_{z}}\left(\partial_{\xi} \partial_{x} G_{k, i}(x, \xi)-\partial_{\xi} \partial_{y} G_{k, i}(y, \xi)\right) \delta_{h}(\xi) d \xi, \quad k=1,2,3
\end{aligned}
$$

Let $x, y \in D, x \neq y$, then using that $1 \leq i \leq 3$ by (4.8b), we have

$$
\begin{aligned}
\frac{\left|\partial_{x} g_{k}(x)-\partial_{y} g_{k}(y)\right|}{|x-y|^{\sigma}} & \leq \max _{\xi \in T_{z}} \frac{\left|\partial_{\xi} \partial_{x} G_{k, i}(x, \xi)-\partial_{\xi} \partial_{y} G_{k, i}(y, \xi)\right|}{|x-y|^{\sigma}}\left\|\delta_{h}\right\|_{L^{1}\left(T_{z}\right)} \\
& \leq C \max _{\xi \in T_{z}}\left(|x-\xi|^{-3-\sigma}+|y-\xi|^{-3-\sigma}\right) \leq C d^{-3-\sigma}, \quad k=1,2,3 .
\end{aligned}
$$

In the last inequality we used that for any $\xi \in T_{z},|x-\xi|,|y-\xi| \geq \frac{d}{2}$, and $\left\|\delta_{h}\right\|_{L^{1}\left(T_{z}\right)} \leq C$. Therefore, taking the supremum over $k$ we can conclude,

$$
\sup _{x, y \in D} \frac{|\nabla \vec{g}(x)-\nabla \vec{g}(y)|}{|x-y|^{\sigma}} \leq C d^{-3-\sigma} .
$$

The proof for $\|\lambda\|_{C^{\sigma}(D)}$ is very similar.

Let $\left(\vec{g}_{h}, \lambda_{h}\right) \in \vec{V}_{h} \times M_{h}$ be the corresponding finite element solution, i.e., the unique solution that satisfies

$$
\begin{aligned}
\left(\nabla \vec{g}_{h}, \nabla \vec{\chi}\right)+\left(\nabla \lambda_{h}, \vec{\chi}\right) & =(\nabla \vec{g}, \nabla \vec{\chi})+(\nabla \lambda, \vec{\chi}), \quad \forall \vec{\chi} \in \vec{V}_{h}, \\
\left(\nabla \cdot \vec{g}_{h}, w\right) & =0, \quad \forall w \in M_{h},
\end{aligned}
$$

and $\lambda_{h} \in L_{\phi}^{2}(\Omega)$. 
We have,

$$
\begin{aligned}
- & \partial_{x_{j}}\left(\vec{u}_{h}\right)_{i}(z)=\left(\vec{u}_{h},\left(\partial_{x_{j}} \delta_{h}\right) \vec{e}_{i}\right) \\
& =\left(\vec{u}_{h},-\Delta \vec{g}+\nabla \lambda\right) \\
& =\left(\nabla \vec{u}_{h}, \nabla \vec{g}\right)+\left(\vec{u}_{h}, \nabla \lambda\right) \\
& =\left(\nabla \vec{u}_{h}, \nabla \vec{g}\right)+\left(\vec{u}_{h}, \nabla \lambda_{h}\right)+\left(\nabla \vec{u}_{h}, \nabla\left(\vec{g}_{h}-\vec{g}\right)\right) \\
& =\left(\nabla \vec{u}_{h}, \nabla \vec{g}_{h}\right) \\
& =\left(\nabla \vec{u}, \nabla \vec{g}_{h}\right)+\left(\nabla\left(p-p_{h}\right), \vec{g}_{h}\right) \\
& =\left(\nabla \vec{u}, \nabla \vec{g}_{h}\right)+\left(\nabla p, \vec{g}_{h}\right) \\
& =\left(\nabla \vec{u}, \nabla\left(\vec{g}_{h}-\vec{g}\right)\right)+(\nabla \vec{u}, \nabla \vec{g})+\left(\nabla p, \vec{g}_{h}-\vec{g}\right)+(\vec{u}, \nabla \lambda) \\
& =\left(\nabla \vec{u}, \nabla\left(\vec{g}_{h}-\vec{g}\right)\right)+(\vec{u},-\Delta \vec{g}+\nabla \lambda)+\left(\vec{g}-\vec{g}_{h}, \nabla p\right) \\
& =\left(\nabla \vec{u}, \nabla\left(\vec{g}_{h}-\vec{g}\right)\right)-\left(\frac{\partial(\vec{u})_{i}}{\partial x_{j}}, \delta_{h}\right)-\left(\nabla \cdot\left(\vec{g}-\vec{g}_{h}\right), p\right) .
\end{aligned}
$$

Taking supremum over all partial derivatives and using that $\left\|\delta_{h}\right\|_{L^{1}(\Omega)} \leq C$, we obtain

$$
\left\|\nabla \vec{u}_{h}\right\|_{L^{\infty}(\Omega)} \leq\left(\|\nabla \vec{u}\|_{L^{\infty}(\Omega)}+\|p\|_{L^{\infty}(\Omega)}\right)\left(C+\left\|\nabla\left(\vec{g}_{h}-\vec{g}\right)\right\|_{L^{1}(\Omega)}\right) .
$$

Thus, in order to show the stability for the velocity, we only need to establish the following result.

Lemma 5.2. There exists a constant $C$ independent of $h$ and $\vec{g}$ such that

$$
\left\|\nabla\left(\vec{g}-\vec{g}_{h}\right)\right\|_{L^{1}(\Omega)} \leq C .
$$

Proof. The proof is based on the ideas developed in papers by Schatz and Wahlbin, e.g., [31, 32, 33. We will break it down into four steps.

Step 1 (Dyadic decomposition). Without loss of generality, we assume that the diameter of $\Omega$ is less than 1 . Put $d_{j}=2^{-j}$ and consider a dyadic decomposition of $\Omega$,

$$
\Omega=\Omega^{*} \cup \bigcup_{j=0}^{J} \Omega_{j}
$$

where

$$
\begin{aligned}
& \Omega^{*}=\{x \in \Omega:|x-z| \leq K h\}, \\
& \Omega_{j}=\left\{x \in \Omega: d_{j+1} \leq|x-z| \leq d_{j}\right\},
\end{aligned}
$$

where $K$ is a sufficiently large constant to be chosen later and $J$ is an integer such that $2^{-(J+1)} \leq K h \leq 2^{-J}$. In the analysis below the generic constants will be denoted by $C$, but we will keep track on the explicit dependence of the constants on $K$.

Using the dyadic decomposition and the Cauchy-Schwarz inequality, we have

$$
\left\|\nabla\left(\vec{g}-\vec{g}_{h}\right)\right\|_{L^{1}(\Omega)} \leq C K^{3 / 2} h^{3 / 2}\left\|\nabla\left(\vec{g}-\vec{g}_{h}\right)\right\|_{L^{2}\left(\Omega^{*}\right)}+C \sum_{j=0}^{J} d_{j}^{3 / 2}\left\|\nabla\left(\vec{g}-\vec{g}_{h}\right)\right\|_{L^{2}\left(\Omega_{j}\right)} .
$$


We start with the first term on the right-hand side. Using the global a priori error estimates Proposition 2.1, approximation properties of $\mathbf{P}$ and $\mathbf{R}(2.5 \mathrm{~d})$, (2.5a), $H^{2}$-regularity (4.6), and (5.2), we have

$$
\begin{aligned}
h^{3 / 2}\left\|\nabla\left(\vec{g}-\vec{g}_{h}\right)\right\|_{L^{2}\left(\Omega^{*}\right)} & \leq h^{3 / 2}\left\|\nabla\left(\vec{g}-\vec{g}_{h}\right)\right\|_{L^{2}(\Omega)} \\
& \leq C h^{3 / 2+1}\left(\|\vec{g}\|_{H^{2}(\Omega)}+\|\lambda\|_{H^{1}(\Omega)}\right) \\
& \leq C h^{5 / 2}\left\|\nabla \delta_{h}\right\|_{L^{2}(T)} \leq C .
\end{aligned}
$$

Thus, we have

$$
\left\|\nabla\left(\vec{g}-\vec{g}_{h}\right)\right\|_{L^{1}(\Omega)} \leq C K^{3 / 2}+\sum_{j=0}^{J} M_{j}, \quad \text { with } \quad M_{j}:=d_{j}^{3 / 2}\left\|\nabla\left(\vec{g}-\vec{g}_{h}\right)\right\|_{L^{2}\left(\Omega_{j}\right)} .
$$

Step 2 (Initial Estimate for $M_{j}$ ). Define the following sets:

$$
\begin{aligned}
\Omega_{j}^{\prime} & =\left\{x \in \Omega: d_{j+2} \leq|x-z| \leq d_{j-1}\right\}, \\
\Omega_{j}^{\prime \prime} & =\left\{x \in \Omega: d_{j+3} \leq|x-z| \leq d_{j-2}\right\}, \\
\Omega_{j}^{\prime \prime \prime} & =\left\{x \in \Omega: d_{j+4} \leq|x-z| \leq d_{j-3}\right\} .
\end{aligned}
$$

Notice that Theorem 2 holds for $A_{1}=\Omega_{j}$ and $A_{2}=\Omega_{j}^{\prime}$ with $d=d_{j}, j=1,2, \ldots, J$. Thus, by the local energy estimate, Theorem 2 , and any $0<\varepsilon<1$,

$$
\begin{aligned}
\left\|\nabla\left(\vec{g}-\vec{g}_{h}\right)\right\|_{L^{2}\left(\Omega_{j}\right) \leq} & C\left(\|\nabla(\vec{g}-\mathbf{P} \vec{g})\|_{L^{2}\left(\Omega_{j}^{\prime}\right)}+\frac{1}{\varepsilon d_{j}}\|\vec{g}-\mathbf{P} \vec{g}\|_{L^{2}\left(\Omega_{j}^{\prime}\right)}+\|\lambda-\mathbf{R} \lambda\|_{L^{2}\left(\Omega_{j}^{\prime}\right)}\right) \\
& +\varepsilon\left\|\nabla\left(\vec{g}-\vec{g}_{h}\right)\right\|_{L^{2}\left(\Omega_{j}^{\prime}\right)}+\frac{C}{\varepsilon d_{j}}\left\|\vec{g}-\vec{g}_{h}\right\|_{L^{2}\left(\Omega_{j}^{\prime}\right)} .
\end{aligned}
$$

First we will treat the first two terms on the right-hand side. By the CauchySchwarz inequality and the approximation result (2.5b), we have,

$$
\begin{aligned}
& \|\nabla(\vec{g}-\mathbf{P} \vec{g})\|_{L^{2}\left(\Omega_{j}^{\prime}\right)}+d_{j}^{-1} \varepsilon^{-1}\|\vec{g}-\mathbf{P} \vec{g}\|_{L^{2}\left(\Omega_{j}^{\prime}\right)} \\
& \leq C d_{j}^{3 / 2}\left(\|\nabla(\vec{g}-\mathbf{P} \vec{g})\|_{L^{\infty}\left(\Omega_{j}^{\prime}\right)}+d_{j}^{-1} \varepsilon^{-1}\|\vec{g}-\mathbf{P} \vec{g}\|_{L^{\infty}\left(\Omega_{j}^{\prime}\right)}\right) \\
& \leq C d_{j}^{3 / 2} h^{\sigma}\left(1+h d_{j}^{-1} \varepsilon^{-1}\right)\|\vec{g}\|_{C^{1+\sigma}\left(\Omega_{j}^{\prime \prime}\right)} .
\end{aligned}
$$

Applying Lemma 5.1 with $D=\Omega_{j}^{\prime \prime}$, we obtain

$$
\|\vec{g}\|_{C^{1+\sigma}\left(\Omega_{j}^{\prime \prime}\right)} \leq C d_{j}^{-3-\sigma} .
$$

Thus, we have shown that

$$
\|\nabla(\vec{g}-\mathbf{P} \vec{g})\|_{L^{2}\left(\Omega_{j}^{\prime}\right)}+d_{j}^{-1} \varepsilon^{-1}\|\vec{g}-\mathbf{P} \vec{g}\|_{L^{2}\left(\Omega_{j}^{\prime}\right)} \leq C\left(1+h d_{j}^{-1} \varepsilon^{-1}\right) d_{j}^{-3 / 2-\sigma} h^{\sigma} .
$$

Similarly, using the Cauchy-Schwarz inequality and the approximation estimate (2.5e), we have

$$
\|\lambda-\mathbf{R} \lambda\|_{L^{2}\left(\Omega_{j}^{\prime}\right)} \leq C d_{j}^{3 / 2}\|\lambda-\mathbf{R} \lambda\|_{L^{\infty}\left(\Omega_{j}^{\prime}\right)} \leq C d_{j}^{3 / 2} h^{\sigma}\|\lambda\|_{C^{\sigma}\left(\Omega_{j}^{\prime \prime}\right)} .
$$

Again applying Lemma 5.1 with $D=\Omega_{j}^{\prime \prime}$, we have

$$
\|\lambda\|_{C^{\sigma}\left(\Omega_{j}^{\prime \prime}\right)} \leq C d_{j}^{-3-\sigma}
$$

and as a result

$$
\|\lambda-\mathbf{R} \lambda\|_{L^{2}\left(\Omega_{j}^{\prime}\right)} \leq C d_{j}^{-3 / 2-\sigma} h^{\sigma} .
$$


To summarize,

$M_{j} \leq C\left(\left(1+h d_{j}^{-1} \varepsilon^{-1}\right)\left(h / d_{j}\right)^{\sigma}+d_{j}^{1 / 2} \varepsilon^{-1}\left\|\vec{g}-\vec{g}_{h}\right\|_{L^{2}\left(\Omega_{j}^{\prime}\right)}+\varepsilon d_{j}^{3 / 2}\left\|\nabla\left(\vec{g}-\vec{g}_{h}\right)\right\|_{L^{2}\left(\Omega_{j}^{\prime}\right)}\right)$.

Next, we will use a duality argument to estimate $\left\|\vec{g}-\vec{g}_{h}\right\|_{L^{2}\left(\Omega_{j}^{\prime}\right)}$.

Step 3 (Duality argument). We have the following representation:

$$
\left\|\vec{g}-\vec{g}_{h}\right\|_{L^{2}\left(\Omega_{j}^{\prime}\right)}=\sup _{\substack{\vec{v} \in C_{c}^{\infty}\left(\Omega_{j}^{\prime}\right) \\\|\vec{v}\|_{L^{2}\left(\Omega_{j}^{\prime}\right)} \leq 1}}\left(\vec{g}-\vec{g}_{h}, \vec{v}\right) .
$$

For each such $\vec{v}$, let $\vec{w}, \varphi$ be the solution of the following problem:

$$
\begin{aligned}
-\Delta \vec{w}+\nabla \varphi & =\vec{v} & & \text { in } \Omega, \\
\nabla \cdot \vec{w} & =0 & & \text { in } \Omega, \\
\vec{w} & =\overrightarrow{0} & & \text { on } \partial \Omega .
\end{aligned}
$$

Thus, using that $(\nabla \cdot \mathbf{P} \vec{w}, \chi)=0$ and $\left(\nabla \cdot\left(\vec{g}-\vec{g}_{h}\right), \chi\right)=0$ for any $\chi \in M_{h}$ and $\nabla \cdot \vec{w}=0$, we have

$$
\begin{aligned}
& \left(\vec{g}-\vec{g}_{h}, \vec{v}\right)=\left(\nabla\left(\vec{g}-\vec{g}_{h}\right), \nabla \vec{w}\right)-\left(\varphi, \nabla \cdot\left(\vec{g}-\vec{g}_{h}\right)\right) \\
& \quad=\left(\nabla\left(\vec{g}-\vec{g}_{h}\right), \nabla(\vec{w}-\mathbf{P} \vec{w})\right)+\left(\nabla\left(\vec{g}-\vec{g}_{h}\right), \nabla \mathbf{P} \vec{w}\right)-\left(\varphi-\mathbf{R} \varphi, \nabla \cdot\left(\vec{g}-\vec{g}_{h}\right)\right) \\
& \quad=\left(\nabla\left(\vec{g}-\vec{g}_{h}\right), \nabla(\vec{w}-\mathbf{P} \vec{w})\right)-\left(\lambda-\lambda_{h}, \nabla \cdot \mathbf{P} \vec{w}\right)-\left(\varphi-\mathbf{R} \varphi, \nabla \cdot\left(\vec{g}-\vec{g}_{h}\right)\right) \\
& \quad=\left(\nabla\left(\vec{g}-\vec{g}_{h}\right), \nabla(\vec{w}-\mathbf{P} \vec{w})\right)-(\lambda-\mathbf{R} \lambda, \nabla \cdot(\mathbf{P} \vec{w}-\vec{w}))-\left(\varphi-\mathbf{R} \varphi, \nabla \cdot\left(\vec{g}-\vec{g}_{h}\right)\right) \\
& \quad:=J_{1}+J_{2}+J_{3} .
\end{aligned}
$$

We split $J_{1}$ into two terms as follows:

$$
J_{1}=\left(\nabla\left(\vec{g}-\vec{g}_{h}\right), \nabla(\vec{w}-\mathbf{P} \vec{w})\right)_{\Omega_{j}^{\prime \prime \prime}}+\left(\nabla\left(\vec{g}-\vec{g}_{h}\right), \nabla(\vec{w}-\mathbf{P} \vec{w})\right)_{\Omega \backslash \Omega_{j}^{\prime \prime \prime}}
$$

First we estimate $\left(\nabla\left(\vec{g}-\vec{g}_{h}\right), \nabla(\vec{w}-\mathbf{P} \vec{w})\right)_{\Omega_{j}^{\prime \prime \prime}}$. By the Cauchy-Schwarz inequality, the global a priori error estimate, and $H^{2}$-regularity we have

$$
\begin{aligned}
\left(\nabla\left(\vec{g}-\vec{g}_{h}\right), \nabla(\vec{w}-\mathbf{P} \vec{w})\right)_{\Omega_{j}^{\prime \prime \prime}} & \leq\left\|\nabla\left(\vec{g}-\vec{g}_{h}\right)\right\|_{L^{2}\left(\Omega_{j}^{\prime \prime \prime}\right)}\|\nabla(\vec{w}-\mathbf{P} \vec{w})\|_{L^{2}(\Omega)} \\
& \leq\left\|\nabla\left(\vec{g}-\vec{g}_{h}\right)\right\|_{L^{2}\left(\Omega_{j}^{\prime \prime \prime}\right)} C h\|\vec{w}\|_{H^{2}(\Omega)} \\
& \leq C h\left\|\nabla\left(\vec{g}-\vec{g}_{h}\right)\right\|_{L^{2}\left(\Omega_{j}^{\prime \prime \prime}\right)} .
\end{aligned}
$$

Next we estimate the second term of $J_{1}$. By the Hölder inequality and (2.5b),

$$
\begin{aligned}
\left(\nabla\left(\vec{g}-\vec{g}_{h}\right), \nabla(\vec{w}-\mathbf{P} \vec{w})\right)_{\Omega \backslash \Omega_{j}^{\prime \prime \prime}} & \leq\left\|\nabla\left(\vec{g}-\vec{g}_{h}\right)\right\|_{L^{1}(\Omega)}\|\nabla(\vec{w}-\mathbf{P} \vec{w})\|_{L^{\infty}\left(\Omega \backslash \Omega_{j}^{\prime \prime \prime}\right)} \\
& \leq\left\|\nabla\left(\vec{g}-\vec{g}_{h}\right)\right\|_{L^{1}(\Omega)} C h^{\sigma}\|\vec{w}\|_{C^{1+\sigma}\left(\Omega \backslash \Omega_{j}^{\prime \prime}\right)} .
\end{aligned}
$$

Since $\Omega \backslash \Omega_{j}^{\prime \prime}$ is separated from $\Omega_{j}^{\prime}$ by at least $d_{j}$, for $x, y \in \Omega \backslash \Omega_{j}^{\prime \prime}$, using (4.8b), we have

$$
\begin{aligned}
\frac{\left|\partial_{x} w_{k}(x)-\partial_{y} w_{k}(y)\right|}{|x-y|^{\sigma}} & \leq \sum_{i=1}^{3} \int_{\Omega_{j}^{\prime}} \frac{\left|\partial_{x} G_{k, i}(x, \xi)-\partial_{y} G_{k, i}(y, \xi)\right|}{|x-y|^{\sigma}}|\vec{v}(\xi)| d \xi \\
& \leq C \max _{\xi \in \Omega_{j}^{\prime}}(|x-\xi|+|y-\xi|)^{-2-\sigma} \int_{\Omega_{j}^{\prime}}|\vec{v}(\xi)| d \xi \\
& \leq C d_{j}^{-2-\sigma} d_{j}^{3 / 2}\|\vec{v}\|_{L^{2}\left(\Omega_{j}^{\prime}\right)} \leq C d_{j}^{-1 / 2-\sigma}, \quad \text { for } k=1,2,3 .
\end{aligned}
$$

Hence,

$$
\|\vec{w}\|_{C^{1+\sigma}\left(\Omega \backslash \Omega_{j}^{\prime \prime}\right)} \leq C d_{j}^{-1 / 2-\sigma}
$$


which implies

$$
\left(\nabla\left(\vec{g}-\vec{g}_{h}\right), \nabla(\vec{w}-\mathbf{P} \vec{w})\right)_{\Omega \backslash \Omega_{j}^{\prime \prime}} \leq C h^{\sigma} d_{j}^{-1 / 2-\sigma}\left\|\nabla\left(\vec{g}-\vec{g}_{h}\right)\right\|_{L^{1}(\Omega)} .
$$

Hence,

$$
J_{1} \leq C h^{\sigma} d_{j}^{-1 / 2-\sigma}\left\|\nabla\left(\vec{g}-\vec{g}_{h}\right)\right\|_{L^{1}(\Omega)}+C h\left\|\nabla\left(\vec{g}-\vec{g}_{h}\right)\right\|_{L^{2}\left(\Omega_{j}^{\prime \prime \prime}\right)} .
$$

Similarly, we can split $J_{3}$ into two terms

$$
J_{3}=-\left(\varphi-\mathbf{R} \varphi, \nabla \cdot\left(\vec{g}-\vec{g}_{h}\right)\right)_{\Omega_{j}^{\prime \prime \prime}}-\left(\varphi-\mathbf{R} \varphi, \nabla \cdot\left(\vec{g}-\vec{g}_{h}\right)\right)_{\Omega \backslash \Omega_{j}^{\prime \prime \prime}} .
$$

By the Cauchy-Schwarz inequality, the global a priori error estimate, and $H^{2}$ regularity (4.6) we have

$$
\begin{aligned}
\left(\varphi-\mathbf{R} \varphi, \nabla \cdot\left(\vec{g}-\vec{g}_{h}\right)\right)_{\Omega_{j}^{\prime \prime \prime}} & \leq\|\varphi-\mathbf{R} \varphi\|_{L^{2}(\Omega)}\left\|\nabla\left(\vec{g}-\vec{g}_{h}\right)\right\|_{L^{2}\left(\Omega_{j}^{\prime \prime \prime}\right)} \\
& \leq C h\|\nabla \varphi\|_{L^{2}(\Omega)}\left\|\nabla\left(\vec{g}-\vec{g}_{h}\right)\right\|_{L^{2}\left(\Omega_{j}^{\prime \prime \prime}\right)} \\
& \leq C h\left\|\nabla\left(\vec{g}-\vec{g}_{h}\right)\right\|_{L^{2}\left(\Omega_{j}^{\prime \prime \prime}\right)} .
\end{aligned}
$$

Next we estimate the second term of $J_{3}$. By the Hölder inequality and (2.5e),

$$
\begin{aligned}
\left(\varphi-\mathbf{R} \varphi, \nabla \cdot\left(\vec{g}-\vec{g}_{h}\right)\right)_{\Omega \backslash \Omega_{j}^{\prime \prime \prime}} & \leq\|\varphi-\mathbf{R} \varphi\|_{L^{\infty}\left(\Omega \backslash \Omega_{j}^{\prime \prime \prime}\right)}\left\|\nabla\left(\vec{g}-\vec{g}_{h}\right)\right\|_{L^{1}(\Omega)} \\
& \leq C h^{\sigma}\|\varphi\|_{C^{\sigma}\left(\Omega \backslash \Omega_{j}^{\prime \prime}\right)}\left\|\nabla\left(\vec{g}-\vec{g}_{h}\right)\right\|_{L^{1}(\Omega)} .
\end{aligned}
$$

Since $\Omega \backslash \Omega_{j}^{\prime \prime}$ is separated from $\Omega_{j}^{\prime}$ by at least $d_{j}$, we have for $x, y \in \Omega \backslash \Omega_{j}^{\prime \prime}$, using (4.8b)

$$
\begin{aligned}
\frac{|\varphi(x)-\varphi(y)|}{|x-y|^{\sigma}} & \leq \sum_{i=1}^{3} \int_{\Omega_{j}^{\prime}} \frac{\left|G_{4, i}(x, \xi)-G_{4, i}(y, \xi)\right|}{|x-y|^{\sigma}}|\vec{v}(\xi)| d \xi \\
& \leq C \max _{\xi \in \Omega_{j}^{\prime}}(|x-\xi|+|y-\xi|)^{-2-\sigma} \int_{\Omega_{j}^{\prime}}|\vec{v}(\xi)| d \xi \\
& \leq C d_{j}^{-2-\sigma} d_{j}^{3 / 2}\|\vec{v}\|_{L^{2}\left(\Omega_{j}^{\prime}\right)} \leq C d_{j}^{-1 / 2-\sigma} .
\end{aligned}
$$

Hence,

$$
\|\varphi\|_{C^{\sigma}\left(\Omega \backslash \Omega_{j}^{\prime \prime}\right)} \leq C d_{j}^{-1 / 2-\sigma}
$$

which implies that

$$
\left(\varphi-\mathbf{R} \varphi, \nabla \cdot\left(\vec{g}-\vec{g}_{h}\right)\right)_{\Omega \backslash \Omega_{j}^{\prime \prime \prime}} \leq C h^{\sigma} d_{j}^{-1 / 2-\sigma}\left\|\nabla\left(\vec{g}-\vec{g}_{h}\right)\right\|_{L^{1}(\Omega)} .
$$

Hence,

$$
J_{3} \leq C h^{\sigma} d_{j}^{-1 / 2-\sigma}\left\|\nabla\left(\vec{g}-\vec{g}_{h}\right)\right\|_{L^{1}(\Omega)}+C h\left\|\nabla\left(\vec{g}-\vec{g}_{h}\right)\right\|_{L^{2}\left(\Omega_{j}^{\prime \prime \prime}\right)} .
$$

Thus, it remains to estimate $J_{2}$. Similarly as above we split it into two terms,

$$
J_{2}=-(\lambda-\mathbf{R} \lambda, \nabla \cdot(\vec{w}-\mathbf{P} \vec{w}))_{\Omega_{j}^{\prime \prime \prime}}-(\lambda-\mathbf{R} \lambda, \nabla \cdot(\vec{w}-\mathbf{P} \vec{w}))_{\Omega \backslash \Omega_{j}^{\prime \prime \prime}}
$$

By the Cauchy-Schwarz inequality, the global a priori error estimate, and $\mathrm{H}^{2}$ regularity (4.6) we have

$$
\begin{aligned}
(\lambda-\mathbf{R} \lambda, \nabla \cdot(\vec{w}-\mathbf{P} \vec{w}))_{\Omega_{j}^{\prime \prime \prime}} & \leq\|\lambda-\mathbf{R} \lambda\|_{L^{2}\left(\Omega_{j}^{\prime \prime}\right)}\|\nabla(\vec{w}-\mathbf{P} \vec{w})\|_{L^{2}(\Omega)} \\
& \leq\|\lambda\|_{L^{2}\left(\Omega_{j}^{\prime \prime \prime}\right)} C h\|\vec{w}\|_{H^{2}(\Omega)} \leq C h\|\lambda\|_{L^{2}\left(\Omega_{j}^{\prime \prime}\right)} .
\end{aligned}
$$


Using (4.8a) and that $\operatorname{dist}\left(\Omega_{j}^{\prime \prime \prime}, T_{z}\right)=O\left(d_{j}\right)$ we have

$$
\begin{aligned}
\lambda(x) & =\sum_{k=1}^{3} \int_{T_{z}} G_{4, k}(x, \xi)\left(\partial_{\xi} \delta_{h}(\xi)\right) \delta_{i, k} d \xi \\
& =-\int_{T_{z}} \partial_{\xi} G_{4, i}(x, \xi) \delta_{h}(\xi) d \xi \leq C d_{j}^{-3}\left\|\delta_{h}\right\|_{L^{1}\left(T_{z}\right)} \leq C d_{j}^{-3} .
\end{aligned}
$$

Thus,

$$
\|\lambda\|_{L^{2}\left(\Omega_{j}^{\prime \prime \prime}\right)} \leq C d_{j}^{-3 / 2}
$$

and

$$
(\lambda-\mathbf{R} \lambda, \nabla \cdot(\vec{w}-\mathbf{P} \vec{w}))_{\Omega_{j}^{\prime \prime \prime}} \leq C h d_{j}^{-3 / 2} .
$$

The second term in $J_{2}$ by the Hölder inequality and (2.5e), we can estimate as

$$
\begin{aligned}
(\lambda-\mathbf{R} \lambda, \nabla(\vec{w}-\mathbf{P} \vec{w}))_{\Omega \backslash \Omega_{j}^{\prime \prime \prime}} & \leq\|\lambda-\mathbf{R} \lambda\|_{L^{1}(\Omega)}\|\nabla(\vec{w}-\mathbf{P} \vec{w})\|_{L^{\infty}\left(\Omega \backslash \Omega_{j}^{\prime \prime \prime}\right)} \\
& \leq C h^{\sigma}\|\vec{w}\|_{C^{1+\sigma}\left(\Omega \backslash \Omega_{j}^{\prime \prime}\right)} .
\end{aligned}
$$

In the last step we used $\|\lambda-\mathbf{R} \lambda\|_{L^{1}(\Omega)} \leq C$, which we will establish in Section 5.1.1. Since

$$
\|\vec{w}\|_{C^{1+\sigma}\left(\Omega \backslash \Omega_{j}^{\prime \prime}\right)} \leq C d_{j}^{-1 / 2-\sigma}
$$

we have

$$
(\lambda-\mathbf{R} \lambda, \nabla \cdot(\vec{w}-\mathbf{P} \vec{w}))_{\Omega \backslash \Omega_{j}^{\prime \prime \prime}} \leq C h^{\sigma} d_{j}^{-1 / 2-\sigma},
$$

and as a result,

$$
J_{2} \leq C h d_{j}^{-3 / 2}+C h^{\sigma} d_{j}^{-1 / 2-\sigma} .
$$

Therefore, estimates for $J_{1}, J_{2}$, and $J_{3}$, (5.12), (5.16) and (5.13), respectively, give

$$
\begin{aligned}
d_{j}^{1 / 2} \varepsilon^{-1}\left\|\vec{g}-\vec{g}_{h}\right\|_{L^{2}\left(\Omega_{j}^{\prime}\right)} \leq C h d_{j}^{-1} \varepsilon^{-1}+C h^{\sigma} d_{j}^{-\sigma} \varepsilon^{-1} \\
\quad+C h^{\sigma} d_{j}^{-\sigma} \varepsilon^{-1}\left\|\nabla\left(\vec{g}-\vec{g}_{h}\right)\right\|_{L^{1}(\Omega)}+C h d_{j}^{1 / 2} \varepsilon^{-1}\left\|\nabla\left(\vec{g}-\vec{g}_{h}\right)\right\|_{L^{2}\left(\Omega_{j}^{\prime \prime \prime}\right)} .
\end{aligned}
$$

To summarize,

$$
\begin{aligned}
M_{j} \leq C\left(\left(1+\varepsilon^{-1}\right)\left(h / d_{j}\right)^{\sigma}+\left(h / d_{j}\right) \varepsilon^{-1}\right. & +\left(h / d_{j}\right)^{\sigma} \varepsilon^{-1}\left\|\nabla\left(g-g_{h}\right)\right\|_{L^{1}(\Omega)} \\
& \left.+\left(h d_{j}^{1 / 2} \varepsilon^{-1}+\varepsilon\right)\left\|\nabla\left(g-g_{h}\right)\right\|_{L^{2}\left(\Omega_{j}^{\prime \prime \prime}\right)}\right) .
\end{aligned}
$$

Step 4 (Double kick-back argument). Summing over $j$ we obtain

$$
\begin{aligned}
\sum_{j=0}^{J} M_{j} \leq \frac{C\left(1+\varepsilon^{-1}\right)}{K^{\sigma}} & +\frac{C \varepsilon^{-1}}{K}+\frac{C \varepsilon^{-1}}{K^{\sigma}}\left\|\nabla\left(\vec{g}-\vec{g}_{h}\right)\right\|_{L^{1}(\Omega)} \\
& +C\left(\frac{h}{d_{J}} \varepsilon^{-1}+\varepsilon\right) \sum_{j=0}^{J} d_{j}^{3 / 2}\left\|\nabla\left(\vec{g}-\vec{g}_{h}\right)\right\|_{L^{2}\left(\Omega_{j}^{\prime \prime \prime}\right)},
\end{aligned}
$$

where we have used that

$$
\sum_{j=0}^{J}\left(h d_{j}^{-1}\right)^{\sigma} \leq h^{\sigma} \sum_{j=0}^{J} 2^{j \sigma} \leq C h^{\sigma} 2^{\sigma J} \leq C K^{-\sigma} \quad \text { and } \quad d_{j}^{-1} \leq d_{J}^{-1} .
$$


Clearly,

$$
\begin{aligned}
\sum_{j=0}^{J} d_{j}^{3 / 2}\left\|\nabla\left(\vec{g}-\vec{g}_{h}\right)\right\|_{L^{2}\left(\Omega_{j}^{\prime \prime \prime}\right)} & \leq C \sum_{j=0}^{J} M_{j}+C(K h)^{3 / 2}\left\|\nabla\left(\vec{g}-\vec{g}_{h}\right)\right\|_{L^{2}\left(\Omega^{*}\right)} \\
& \leq C \sum_{j=0}^{J} M_{j}+C K^{3 / 2} .
\end{aligned}
$$

Thus, using that $h / d_{J} \leq K^{-1}$, and taking $K$ large enough and $\varepsilon$ small enough, we have

$$
\sum_{j=0}^{J} M_{j} \leq C_{K, \varepsilon}+\frac{C \varepsilon^{-1}}{K^{\sigma}}\left\|\nabla\left(\vec{g}-\vec{g}_{h}\right)\right\|_{L^{1}(\Omega)} .
$$

Therefore, if we plug this result into (5.6) we get

$$
\left\|\nabla\left(\vec{g}-\vec{g}_{h}\right)\right\|_{L^{1}(\Omega)} \leq C_{K, \varepsilon}+\frac{C \varepsilon^{-1}}{K^{\sigma}}\left\|\nabla\left(\vec{g}-\vec{g}_{h}\right)\right\|_{L^{1}(\Omega)} .
$$

Again by choosing $K$ large enough we can conclude

$$
\left\|\nabla\left(\vec{g}-\vec{g}_{h}\right)\right\|_{L^{1}(\Omega)} \leq C_{K, \varepsilon} .
$$

Thus the proof of Lemma (5.2) is complete.

5.1.1. Estimate of $\|\lambda-\mathbf{R} \lambda\|_{L^{1}(\Omega)}$. In this section we prove the following result.

Lemma 5.3. There exists a constant $C$ independent of $h$ and $\lambda$ such that

$$
\|\lambda-\mathbf{R} \lambda\|_{L^{1}(\Omega)} \leq C .
$$

Proof. Using the dyadic decomposition defined in (5.5) and the Cauchy-Schwarz inequality, we have

$$
\|\lambda-\mathbf{R} \lambda\|_{L^{1}(\Omega)} \leq C K^{3 / 2} h^{3 / 2}\|\lambda-\mathbf{R} \lambda\|_{L^{2}\left(\Omega^{*}\right)}+C \sum_{j=0}^{J} d_{j}^{3 / 2}\|\lambda-\mathbf{R} \lambda\|_{L^{2}\left(\Omega_{j}\right)} .
$$

Using the approximation property of $\mathbf{R}(2.5 \mathrm{~d}), H^{2}$-regularity (4.6), and (5.2), we have

$$
h^{3 / 2}\|\lambda-\mathbf{R} \lambda\|_{L^{2}\left(\Omega^{*}\right)} \leq C h^{3 / 2+1}\|\nabla \lambda\|_{L^{2}(\Omega)} \leq C h^{5 / 2}\left\|\nabla \delta_{h}\right\|_{L^{2}(T)} \leq C .
$$

In (5.9) we already established that

$$
\|\lambda-\mathbf{R} \lambda\|_{L^{2}\left(\Omega_{j}\right)} \leq C d_{j}^{-3 / 2-\sigma} h^{\sigma},
$$

hence,

$$
\|\lambda-\mathbf{R} \lambda\|_{L^{1}(\Omega)} \leq C K^{3 / 2}+C \sum_{j=0}^{J} d_{j}^{-\sigma} h^{\sigma} \leq C_{K}
$$


5.2. Part 2, Stability for pressure. Our goal is to show that there exists a constant $C$ independent of $p$ and $h$ such that

$$
\left\|p_{h}\right\|_{L^{\infty}(\Omega)} \leq C\left(\|p\|_{L^{\infty}(\Omega)}+\|\nabla \vec{u}\|_{L^{\infty}(\Omega)}\right) .
$$

Let $z \in T_{z}$ be such that $\left\|p_{h}\right\|_{L^{\infty}(\Omega)}=\left|p_{h}(z)\right|$. Let $\delta_{h}$ be a smooth delta function defined in (5.1). Define a pair $(\vec{\Theta}, \Sigma) \in H_{0}^{1}(\Omega)^{3} \times L_{\phi}^{2}(\Omega)$ by the equation

$$
\begin{aligned}
-\Delta \vec{\Theta}+\nabla \Sigma & =0 & & \text { in } \Omega, \\
\nabla \cdot \vec{\Theta} & =\delta_{h}-\phi & & \text { in } \Omega, \\
\vec{\Theta} & =0 & & \text { on } \partial \Omega .
\end{aligned}
$$

Note that (2.3) implies that $\int_{\Omega}\left(\delta_{h}(x)-\phi(x)\right) d x=0$. Then,

$$
p_{h}(z)=\left(p_{h}, \delta_{h}\right)=\left(p_{h}, \delta_{h}-\phi\right)+\left(p_{h}, \phi\right) .
$$

The second term on the right-hand side of (5.19) can be estimated by using the Cauchy-Schwarz inequality and the a priori error estimate from Proposition 2.1 as

$$
\begin{aligned}
\left(p_{h}, \phi\right) & =\left(p_{h}-p, \phi\right)+(p, \phi) \\
& \leq C\left(\left\|p-p_{h}\right\|_{L^{2}(\Omega)}+\|p\|_{L^{2}(\Omega)}\right)\|\phi\|_{L^{2}(\Omega)} \\
& \leq C\left(\|\nabla \vec{u}\|_{L^{2}(\Omega)}+\|p\|_{L^{2}(\Omega)}\right) \\
& \leq C\left(\|\nabla \vec{u}\|_{L^{\infty}(\Omega)}+\|p\|_{L^{\infty}(\Omega)}\right) .
\end{aligned}
$$

To estimate the first term on the right-hand side of (5.19) we use (5.18), property of $\mathbf{P}$, namely (2.4), to obtain

$$
\begin{aligned}
\left(p_{h}, \delta_{h}-\phi\right) & =\left(p_{h}, \nabla \cdot \vec{\Theta}\right)=\left(p_{h}, \nabla \cdot \mathbf{P} \vec{\Theta}\right) \\
& =(p, \nabla \cdot \mathbf{P} \vec{\Theta})+\left(p_{h}-p, \nabla \cdot \mathbf{P} \vec{\Theta}\right):=I_{1}+I_{2} .
\end{aligned}
$$

Using (5.18), the Hölder inequality, and the properties of $\phi$ and $\delta_{h}$ we have,

$$
\begin{aligned}
I_{1} & =(p, \nabla \cdot(\mathbf{P} \vec{\Theta}-\vec{\Theta}))+(p, \nabla \cdot \vec{\Theta}) \\
& =(p, \nabla \cdot(\mathbf{P} \vec{\Theta}-\vec{\Theta}))+\left(p, \delta_{h}-\phi\right) \\
& \leq\|p\|_{L^{\infty}(\Omega)}\left(\|\nabla(\mathbf{P} \vec{\Theta}-\vec{\Theta})\|_{L^{1}(\Omega)}+\|\phi\|_{L^{1}(\Omega)}+\|\delta\|_{L^{1}(\Omega)}\right) \\
& \leq\|p\|_{L^{\infty}(\Omega)}\left(\|\nabla(\mathbf{P} \vec{\Theta}-\vec{\Theta})\|_{L^{1}(\Omega)}+C\right) .
\end{aligned}
$$

To estimate $I_{2}$ we use orthogonality of $\vec{u}-\vec{u}_{h}$ and (5.18) to obtain,

$$
\begin{aligned}
I_{2} & =\left(\nabla\left(\vec{u}-\vec{u}_{h}\right), \nabla \mathbf{P} \vec{\Theta}\right)=\left(\nabla\left(\vec{u}-\vec{u}_{h}\right), \nabla \vec{\Theta}\right)+\left(\nabla\left(\vec{u}-\vec{u}_{h}\right), \nabla(\mathbf{P} \vec{\Theta}-\vec{\Theta})\right) \\
& =-\left(\Sigma, \nabla \cdot\left(\vec{u}-\vec{u}_{h}\right)\right)+\left(\nabla\left(\vec{u}-\vec{u}_{h}\right), \nabla(\mathbf{P} \vec{\Theta}-\vec{\Theta})\right) \\
& =-\left(\Sigma-\mathbf{R} \Sigma, \nabla \cdot\left(\vec{u}-\vec{u}_{h}\right)\right)+\left(\nabla\left(\vec{u}-\vec{u}_{h}\right), \nabla(\mathbf{P} \vec{\Theta}-\vec{\Theta})\right) \\
& \leq\left\|\nabla\left(\vec{u}-\vec{u}_{h}\right)\right\|_{L^{\infty}(\Omega)}\left(\|\nabla(\mathbf{P} \vec{\Theta}-\vec{\Theta})\|_{L^{1}(\Omega)}+\|\Sigma-\mathbf{R} \Sigma\|_{L^{1}(\Omega)}\right) .
\end{aligned}
$$

Since we have already estimated $\left\|\nabla\left(\vec{u}-\vec{u}_{h}\right)\right\|_{L^{\infty}(\Omega)}$, to obtain the desired estimate of the error for the pressure we need to establish

Lemma 5.4.

$$
\|\nabla(\mathbf{P} \vec{\Theta}-\vec{\Theta})\|_{L^{1}(\Omega)}+\|\Sigma-\mathbf{R} \Sigma\|_{L^{1}(\Omega)} \leq C .
$$


Proof. Using the dyadic decomposition (5.5) and the triangle inequality we have $\|\nabla(\mathbf{P} \vec{\Theta}-\vec{\Theta})\|_{L^{1}(\Omega)}+\|\Sigma-\mathbf{R} \Sigma\|_{L^{1}(\Omega)} \leq\|\nabla(\mathbf{P} \vec{\Theta}-\vec{\Theta})\|_{L^{1}\left(\Omega_{*}\right)}+\|\Sigma-\mathbf{R} \Sigma\|_{L^{1}\left(\Omega_{*}\right)}$

$$
+\sum_{j=1}^{J}\left(\|\nabla(\mathbf{P} \vec{\Theta}-\vec{\Theta})\|_{L^{1}\left(\Omega_{j}\right)}+\|\Sigma-\mathbf{R} \Sigma\|_{L^{1}\left(\Omega_{j}\right)}\right) .
$$

First we will estimate the errors over the innermost subdomain. By CauchySchwartz inequality, approximation properties (2.5d) and (2.5a), and $H^{2}$-regularity (4.6) we have

$$
\begin{aligned}
\|\nabla(\mathbf{P} \vec{\Theta}-\vec{\Theta})\|_{L^{1}\left(\Omega_{*}\right)} & +\|\Sigma-\mathbf{R} \Sigma\|_{L^{1}\left(\Omega_{*}\right)} \\
& \leq C K^{3 / 2} h^{3 / 2}\left(\|\nabla(\mathbf{P} \vec{\Theta}-\vec{\Theta})\|_{L^{2}(\Omega)}+\|\Sigma-\mathbf{R} \Sigma\|_{L^{2}(\Omega)}\right) \\
& \leq C K^{3 / 2} h^{3 / 2+1}\left(\|\vec{\Theta}\|_{H^{2}(\Omega)}+\|\Sigma\|_{H^{1}(\Omega)}\right) \\
& \leq C K^{3 / 2} h^{3 / 2+1}\left(\left\|\delta_{h}\right\|_{H^{1}(\Omega)}+\|\phi\|_{H^{1}(\Omega)}\right) \leq C .
\end{aligned}
$$

To estimate the terms over $\Omega_{j}$ we use the Hölder inequality and the approximation theory to obtain

$$
\begin{aligned}
\|\nabla(\mathbf{P} \vec{\Theta}-\vec{\Theta})\|_{L^{1}\left(\Omega_{j}\right)} & +\left\|\Sigma_{1}-\mathbf{R} \Sigma_{1}\right\|_{L^{1}\left(\Omega_{j}\right)} \\
& \leq C d_{j}^{3}\left(\|\nabla(\mathbf{P} \vec{\Theta}-\vec{\Theta})\|_{C\left(\Omega_{j}\right)}+\|\Sigma-\mathbf{R} \Sigma\|_{C\left(\Omega_{j}\right)}\right) \\
& \leq C h^{\sigma} d_{j}^{3}\left(\|\vec{\Theta}\|_{C^{1+\sigma}\left(\Omega_{j}^{\prime}\right)}+\|\Sigma\|_{C^{\sigma}\left(\Omega_{j}^{\prime}\right)}\right) .
\end{aligned}
$$

By the Green's matrix representation (4.7a) and (4.7b), and using (4.4), we have

$$
(\vec{\Theta})_{i}(x)=\int_{\Omega} G_{i, 4}(x, \xi) \delta_{h}(\xi) d \xi, \quad i=1,2,3
$$

and

$$
\Sigma(x)=\int_{\Omega} G_{4,4}(x, \xi) \delta_{h}(\xi) d \xi .
$$

Using the above representation, (4.8b), and the fact that $\operatorname{dist}\left(T_{z}, \Omega_{j}^{\prime}\right)=O\left(d_{j}\right)$ we obtain

$$
\begin{aligned}
\frac{\partial_{x}(\vec{\Theta})_{i}(x)-\partial_{y}(\vec{\Theta})_{i}(y)}{|x-y|^{\sigma}} & =\int_{T_{z}} \frac{\partial_{x} G_{i, 4}(x, \xi)-\partial_{y} G_{i, 4}(y, \xi)}{|x-y|^{\sigma}} \delta_{h}(\xi) d \xi \\
& \leq C \max _{\xi \in T_{z}}\left(|x-\xi|^{-3-\sigma}+|y-\xi|^{-3-\sigma}\right) \leq C d_{j}^{-3-\sigma}
\end{aligned}
$$

Similarly,

$$
\begin{aligned}
\frac{\Sigma(x)-\Sigma(y)}{|x-y|^{\sigma}} & =\int_{T_{z}} \frac{G_{4,4}(x, \xi)-G_{4,4}(y, \xi)}{|x-y|^{\sigma}} \delta_{h}(\xi) d \xi \\
& \leq C \max _{\xi \in T_{z}}\left(|x-\xi|^{-3-\sigma}+|y-\xi|^{-3-\sigma}\right) \leq C d_{j}^{-3-\sigma} .
\end{aligned}
$$

Hence the sum in (5.20) can be bounded as

$$
\begin{aligned}
& \sum_{j=1}^{J}\left(\|\nabla(\mathbf{P} \vec{\Theta}-\vec{\Theta})\|_{L^{1}\left(\Omega_{j}\right)}+\|\Sigma-\mathbf{R} \Sigma\|_{L^{1}\left(\Omega_{j}\right)}\right) \\
& \quad \leq C \sum_{j=1}^{J} h^{\sigma} d_{j}^{3}\left(\|\vec{\Theta}\|_{C^{1+\sigma}\left(\Omega_{j}^{\prime}\right)}+\|\Sigma\|_{C^{\sigma}\left(\Omega_{j}^{\prime}\right)}\right) \leq C \sum_{j=1}^{J} h^{\sigma} d_{j}^{-\sigma} \leq C .
\end{aligned}
$$

Thus we have established Lemma [5.4, (5.17), and as a result, Theorem 1. 


\section{Extensions AND OPEN PROBLEMS.}

In this section we briefly comment on possible extensions and some open problems.

6.1. Localized estimates. In [30], pointwise error estimates having a sharply local character for scalar second order elliptic equations were proved. In the following publications such localized estimates were established for mixed methods [6], discontinuous Galerkin methods [2, 15, parabolic problems [19], and the Stokes problem on smooth domains [3, 16, and for a posteriori error estimates [7. The main result in [3] essentially says that when $\partial \Omega$ is smooth and certain assumptions are satisfied, then for any $z \in \Omega$, the following estimate holds,

$$
\begin{aligned}
& \left|\nabla\left(\vec{u}-\vec{u}_{h}\right)(z)\right|+\left|\left(p-p_{h}\right)(z)\right| \\
& \quad \leq C \ell_{h, s} \min _{(\vec{\chi}, w) \in\left(\vec{V}_{h}, M_{h}\right)}\left(\|\vec{u}-\vec{\chi}\|_{W_{\infty}^{1}(\Omega), \sigma, s}+\|p-w\|_{L^{\infty}(\Omega), \sigma, s}\right),
\end{aligned}
$$

where $\|\cdot\|_{W_{\infty}^{1}(\Omega), \sigma, s}$ and $\|\cdot\|_{L^{\infty}(\Omega), \sigma, s}$ are weighted Sobolev norms with weight function $\sigma_{z}^{s}(y)=\left(\frac{h}{h+|z-y|}\right)^{s}$. Here $0 \leq s \leq k$ and $\ell_{h, s}$ is a logarithmic factor which is needed when $s=k$. In [17] it was remarked that similar localized estimates hold for convex polyhedral domains for second-order problems as well, except that the allowed range of $s$ above is restricted by the maximum interior angle of $\partial \Omega$ as well as by the polynomial degree $k$. It is possible to prove a similar result here. In particular, (6.1) holds for a similar range of $s$. The proof of (6.1) for convex polyhedra may be accomplished by following the current proof with factoring the weight function from the terms in the dyadic decomposition and a careful bookkeeping.

6.2. Graded meshes. Our result, like most results on finite element estimates in maximum norm, assumes that the mesh is quasi-uniform. However, in [8] the stability of the Ritz projection in $W_{\infty}^{1}$ norm was established for more general graded meshes, that hold in most adaptive codes. The essential part of the proof was interior error estimates in $W_{\infty}^{1}$ norm. Such interior error estimates were established for the second order elliptic equations for quasi-uniform meshes away from the boundary in 33 , but for the Stokes problems such estimates are not known. The only result in this direction is [26], which establishes maximum-norm interior error estimates for stable finite element approximations of the Stokes equations in the case of translation invariant meshes.

\section{ACKNOWLEDGEMENTS}

We are indebted to Jürgen Rossmann for many discussions on Green's function estimates. We would also like to thank Alan Demlow and Hongjie Dong for valuable discussions and the anonymous referee for helping to improve the presentation of the paper.

\section{REFERENCES}

[1] D. N. ARnold And X. B. Liu, Local error estimates for finite element discretizations of the Stokes equations, RAIRO Modél. Math. Anal. Numér., 29 (1995), pp. 367-389. MR1342712 (96d:76055) 
[2] H. Chen, Pointwise error estimates of the local discontinuous Galerkin method for a second order elliptic problem, Math. Comp., 74 (2005), pp. 1097-1116 (electronic). MR 2136995 (2006b:65166)

[3] - Pointwise error estimates for finite element solutions of the Stokes problem, SIAM J. Numer. Anal., 44 (2006), pp. 1-28 (electronic). MR2217368(2007a:65190)

[4] M. DAuge, Stationary Stokes and Navier-Stokes systems on two- or three-dimensional domains with corners. I. Linearized equations, SIAM J. Math. Anal., 20 (1989), pp. 74-97. MR.977489(90b:35191)

[5] J. C. De los Reyes, C. Meyer, And B. Vexler, Finite element error analysis for stateconstrained optimal control of the Stokes equations, Control Cybernet., 37 (2008), pp. 251284. MR2472877(2009k:49072)

[6] A. DemLow, Localized pointwise a posteriori error estimates for gradients of piecewise linear finite element approximations to second-order quadilinear elliptic problems, SIAM J. Numer. Anal., 44 (2006), pp. 494-514 (electronic). MR2218957 (2007c:65105)

[7] A. Demlow and S. Larsson, Local pointwise a posteriori gradient error bounds for the Stokes equation, (submitted).

[8] A. Demlow, D. Leykekhman, A. Schatz, and L. Wahlbin, Best approximation property in the $W_{\infty}^{1}$ norm on graded meshes, Math. Comp., 81 (2012) pp. 743-764.

[9] R. Durán, R. H. Nochetto, And J. P. WAng, Sharp maximum norm error estimates for finite element approximations of the Stokes problem in 2-D, Math. Comp., 51 (1988), pp. 491-506. MR.935076(89b:65261)

[10] A. ERn And J.-L. Guermond, Theory and practice of finite elements, vol. 159 of Applied Mathematical Sciences, Springer-Verlag, New York, 2004. MR2050138(2005d:65002)

[11] G. P. Galdi, An Introduction to the Mathematical Theory of the Navier-Stokes Equations. Volume I. Linearized Steady Problems, Springer Tracts in Natural Philosophy, Vol. 38, Springer-Verlag, Berlin, Heidelberg, New York, 1994. MR1284205 (95i:35216a)

[12] M. Giaquinta And G. Modica, Nonlinear systems of the type of the stationary Navier-Stokes system, J. Reine Angew. Math., 330 (1982), pp. 173-214. MR641818 (83h:35041)

[13] V. Girault, R. H. Nochetto, and R. Scott, Maximum-norm stability of the finite element Stokes projection, J. Math. Pures Appl. (9), 84 (2005), pp. 279-330. MR2121575 (2006j:76087)

[14] V. Girault And L. R. SCOTt, A quasi-local interpolation operator preserving the discrete divergence, Calcolo, 40 (2003), pp. 1-19. MR.1961943 (2004a:65151)

[15] J. Guzmán, Pointwise error estimates for discontinuous Galerkin methods with lifting operators for elliptic problems, Math. Comp., 75 (2006), pp. 1067-1085 (electronic). MR2219019 $(2006 \mathrm{~m}: 65269)$

[16] Local and pointwise error estimates of the local discontinuous Galerkin method applied to the Stokes problem, Math. Comp., 77 (2008), pp. 1293-1322. MR2398769 (2009d:65171)

[17] J. Guzmán, D. Leykekhman, J. Rossmann, and A. H. Schatz, Hölder estimates for Green's functions on convex polyhedral domains and their applications to finite element methods, Numer. Math., 112 (2009), pp. 221-243. MR2495783(2010a:65237)

[18] V. A. Kozlov, V. G. Maz'ya, and J. Rossmann, Spectral problems associated with corner singularities of solutions to elliptic equations, vol. 85 of Mathematical Surveys and Monographs, American Mathematical Society, Providence, RI, 2001. MR1788991 (2001i:35069)

[19] D. Leykekhman, Pointwise localized error estimates for parabolic finite element equations, Numer. Math., 96 (2004), pp. 583-600. MR2028727 (2004k:65175)

[20] V. MaZ'ya And J. Rossmann, Pointwise estimates for Green's kernel of a mixed boundary value problem to the Stokes system in a polyhedral cone, Math. Nachr., 278 (2005), pp. 17661810. MR2182091 (2007b:35269)

[21] V. MaZ'ya AND J. Rossmann, $L_{p}$ estimates of solutions to mixed boundary value problems for the Stokes system in polyhedral domains, Math. Nachr., 280 (2007), pp. 751-793. MR2321139 (2008m:35280)

[22] V. MAZ'YA AND J. Rossmann, Elliptic equations in polyhedral domains, vol. 162 of Mathematical Surveys and Monographs, American Mathematical Society, Providence, RI, 2010. MR2641539(2011h:35002) 
[23] V. G. MaZ'YA And B. A. Plamenevskil̆, The first boundary value problem for classical equations of mathematical physics in domains with piecewise-smooth boundaries. I, Z. Anal. Anwendungen, 2 (1983), pp. 335-359. MR725151 (86c:35123)

[24] . The first boundary value problem for classical equations of mathematical physics in domains with piecewise smooth boundaries. II, Z. Anal. Anwendungen, 2 (1983), pp. 523-551. MR734895 (86c:35124)

[25] V. G. MAZ'YA AND J. Rossmann, Schauder estimates for solutions to a mixed boundary value problem for the Stokes system in polyhedral domains, Math. Methods Appl. Sci., 29 (2006), pp. 965-1017. MR2228352(2007f:35220)

[26] R. NARASIMHAN AND I. BABUŠKA, Interior maximum norm estimates for finite element discretizations of the Stokes equations, Appl. Anal., 86 (2007), pp. 251-260. MR2297317 (2008c:65343)

[27] J. A. Nitsche And A. H. Schatz, Interior estimates for Ritz-Galerkin methods, Math. Comp., 28 (1974), pp. 937-958. MR0373325(51:9525)

[28] J. Rossmann, Green's matrix of the Stokes system in a convex polyhedron, Rostock. Math. Kolloq., 65 (2010), pp. 1-14. MR2808700

[29] _ Hölder estimates for Green's matrix of the Stokes system in convex polyhedra, in Around the research of Vladimir Maz'ya. II, vol. 12 of Int. Math. Ser. (N. Y.), Springer, New York, 2010, pp. 315-336. MR2676181

[30] A. H. Schatz, Pointwise error estimates and asymptotic error expansion inequalities for the finite element method on irregular grids. I. Global estimates, Math. Comp., 67 (1998), pp. 877-899. MR $1464148(98 \mathrm{j}: 65082)$

[31] A. H. SChATZ AND L. B. WAHLBIN, Interior maximum norm estimates for finite element methods, Math. Comp., 31 (1977), pp. 414-442. MR0431753(55:4748)

[32] - On the quasi-optimality in $L_{\infty}$ of the $\dot{H}^{1}$-projection into finite element spaces, Math. Comp., 38 (1982), pp. 1-22. MR637283 (82m:65106)

[33] _ Interior maximum-norm estimates for finite element methods. II, Math. Comp., 64 (1995), pp. 907-928. MR:1297478 (95j:65143)

Division of Applied Mathematics, Brown University, Providence, Rhode Island 02906 E-mail address: Johnny_Guzman@brown.edu

Department of Mathematics, University of Connecticut, Storrs, Connecticut 06269 E-mail address: leykekhman@math.uconn.edu 